\title{
Valoración de Bases de Datos de Usos de Suelo para la localización y distribución espacial de la energía solar y eólica en España
}

\section{An assessment of Land Use Data Bases for location and spatial distribution of solar and wind energy in Spain}

\author{
Jaime Díaz Pacheco ${ }^{1}$, Richard Hewitt² ${ }^{2}$ Abel López Díez ${ }^{1}$, Pedro Dorta Antequera ${ }^{1}$
}

\begin{abstract}
Resumen
La implantación de energías renovables es una de las acciones más destacadas que se insertan dentro de las políticas de reducción de gases de efecto invernadero. Este es uno de los factores globales de mayor relevancia de su implantación. Sin embargo, desde la perspectiva territorial y local existen otros factores, como la relación con el paisaje, los usos de suelo, el medio ambiente y las actividades socio-económicas, que hacen necesario desarrollar fuentes de información espacial, que permitan conocer y entender las características y el modo a través del cual se implantan las energías renovables. Este trabajo tiene el objetivo de valorar las posibilidades que brinda el 'Sistema de Información de Ocupación del Suelo en España' (SIOSE) para realizar diversos análisis relacionados con los patrones de localización de las instalaciones de energía solar y eólica en el territorio español peninsular. Para llevar a cabo dicha valoración, se analiza el grado de implantación de las instalaciones de producción energética eólica y solar, así como de su distribución por regiones. Al mismo tiempo y, en combinación con la fuente CORINE Land Cover, se distinguen que usos y cobertura de suelo están siendo ocupados por estas energías, y que usos urbanos se encuentran más próximos a las instalaciones de producción. Por último, utilizando información altitudinal y bases de datos de espacios naturales protegidos, se observa como existen diferencias importantes entre la energía solar y eólica en el grado de ocupación de suelo dentro de estos espacios.
\end{abstract}

Palabras clave: CORINE Land Cover, energías renovables, SIOSE, usos de suelo, territorio.

\begin{abstract}
The implementation of renewable energies is one of the most outstanding actions that are included in the policies of reduction of greenhouse gases. This is one of the most important global factors of its implementation. However, from a territorial and local perspective, there are other factors, such as the relationship with the landscape, land uses, the environment and socio-economic activities, which make it necessary to develop spatial information sources, characteristics and the way in which renewable energies are implemented. This work has the objective of assessing the possibilities offered by the 'Land Use Information System in Spain' (SIOSE) to carry out various analyses related to the location patterns of solar and wind energy installations in the Spanish mainland. In order to make the assessment, the degree of implantation of wind and solar energy production facilities is analysed, as well as their distribution by regions. At the same time and in combination with the CORINE Land Cover source, it is clear that land use and land cover are being occupied by these energies, and that urban uses are closer to the production facilities. Finally, using altitudinal information and databases of protected natural spaces, it is observed that there are important differences between solar and wind energy in the degree of soil occupation within these spaces.
\end{abstract}

Keywords: CORINE Land Cover, landscape, land uses, renewable energy, SIOSE, territory.

Recibido el 24 de octubre de 2018, aceptado el 21 de noviembre de 2018.

Cómo citar: Díaz Pacheco, J., Hewitt R., López Diez, A., Dorta Antequera, P. (2018). Valoración de Bases de Datos de Usos de Suelo para la localización y distribución espacial de la energía solar y eólica en España. Investigaciones Geográficas, 56, 114-137. https://doi.org/10.5354/0719-5370.2018.51333

1 Cátedra Universitaria Reducción del Riesgo de Desastres y Ciudades Resilientes. Universidad de La Laguna, San Cristóbal de La Laguna, España. E-mail: jdiazpac@ull.edu.es

2 The James Hutton Institute, Craigiebuckler, Aberdeen AB15 8QH, Scotland UK.

Este trabajo se enmarca en el proyecto COMPLEX del séptimo marco de la Comisión Eurropea. 


\section{Introducción}

Dentro de los esfuerzos globales para mitigar el Cambio Climático, destacan aquellos que se enmarcan en políticas de transición hacia economías de baja producción de carbono. De este modo, son múltiples los marcos internacionales que abordan esta cuestión, desde el último informe del IPCC en 2013-14 (Intergovernmental Panel on Climate Change) hasta el reciente Acuerdo de París en 2015. En esos documentos se incide en la importancia de la descarbonización a través de las energías renovables. Además, la Unión Europea señala ésta, como una de sus prioridades en su estrategia para 2050 (Comisión Europea, 2011). Dentro de estas políticas de transición, se encuentran las energías renovables solar y eólica. En este sentido, el estudio de la evolución del desarrollo y la implementación de este tipo de generación de energías renovables (solar y eólica) en el territorio, adquiere una gran importancia.

Estos estudios se llevan a cabo desde muy diversas ópticas, entre las que encontramos ejemplos que analizan la evolución de la producción y/o el consumo de energía eólica y solar, diferenciando regiones geográficas a diferentes escalas (JägerWaldau, 2007; Chang, Leunge, Wu, \& Yuan, 2003); o publicaciones que demuestran que existe preocupación científica por la sostenibilidad e impacto paisajístico, ambiental y económico de este tipo de energías (Larsen \& Madsen, 2000; Evans, Strezov, \& Evans, 2009; Ignateva \& Pérez, 2008; Ignateva, 2010; Sánchez, García, \& Saguar, 2011); así como trabajos que tratan de analizar la complejidad de carácter socioeconómico de la implementación territorial de las mismas (Zografos \& Saladié, 2012; Alonso et al., 2016). También, dentro de las aproximaciones de tipo espacial o geográfico, se encuentran multitud de ejemplos de análisis de delimitación de grados de potencialidad para la instalación de energía solar y eólica (Amarante et al., 2001; Espejo, 2004; Sözen, Arcaklioğlu, \& Özalp, 2004; González- Ávila, Beltrán-Morales, Troyo-
Diéguez, \& Ortega-Rubio, 2006; Almonacid \& Nahuelhual, 2009; Celik, Muneer, \& Clarke, 2009). Por último, y con mayor relación con el presente trabajo, encontramos estudios cuya preocupación directa es la localización de este tipo de instalaciones y su vínculo con el paisaje que están produciendo en el territorio, y específicamente en España como caso de investigación dentro de la Unión Europea. De este modo, hallamos acercamientos a este campo a través del análisis de distribución en el territorio de las instalaciones solares térmicas y las campos de generadores eólicos, donde los diferentes autores afirman que "el desarrollo de las energías renovables les parece uno de los cambios paisajísticos más importantes en España, junto a la urbanización masiva" (Sánchez et al., 2011).

Las mencionadas aproximaciones de manera general, pero sobre todo aquellas que requieren de análisis geográfico, demandan fuentes de información que permitan localizar las instalaciones energéticas, conocer que suelos están siendo ocupados, que patrones de emplazamiento pueden estar siguiendo, cuál es su extensión y qué relaciones espaciales pueden tener con otras actividades, como la urbanización, los espacios naturales protegidos, etc. De hecho, como cualquier actividad económica que se instala en el territorio, siempre produce impactos que van más allá de la propia ocupación del suelo, como pueden ser la necesidad de instalación de redes eléctricas o la demanda por ejemplo de recursos hídricos (Espejo, 2010).

El Sistema de Información de Ocupación del Suelo en España (SIOSE) está conformado por una base de datos espaciales de usos y cobertura de suelo, que de manera oportuna, cuenta entre sus categorías de clasificación de usos de suelo, con una identificación de superficies dedicadas a instalaciones (parques solares o eólicos) para la generación de energía solar y eólica, reflejando, además, aquellas que se encuentran en construcción. 


\section{Objetivos}

Este trabajo tiene el objetivo de explorar y valorar las posibilidades que brinda la información espacial, en este caso SIOSE, para realizar diversos análisis relacionados con los patrones de localización de este tipo de instalaciones energéticas en el territorio español peninsular. Para ello se realiza un recuento por Comunidades Autónomas de las hectáreas dedicadas a emplazar estas instalaciones para los años de referencia de esta base de datos (2005 y 2011).

Este trabajo tiene el objetivo de explorar y valorar las posibilidades que brinda la información espacial, en este caso SIOSE, para realizar diversos análisis relacionados con los patrones de localización de este tipo de instalaciones energéticas en el territorio español peninsular. Para ello se realiza un recuento por Comunidades Autónomas de las hectáreas dedicadas a emplazar estas instalaciones para los años de referencia de esta base de datos (2005 y 2011).

Se valora también la posibilidad de combinar el uso de SIOSE con una de las bases de datos de usos y cobertura de suelo en Europa, como es CORINE Land Cover (CLC). La evaluación se lleva a cabo analizando territorialmente y por Comunidad Autónoma, sobre qué usos y cobertura de suelo (CLC 2006), se están emplazando estas instalaciones de generación de energía renovable (SIOSE, 2011).

De manera adicional, para valorar la combinación de SIOSE con otras variables del terreno, se exploran los patrones de localización con referencia a variables que puede aportar un modelo digital de elevaciones, como la altitud. Además, se explora el uso de estas bases de datos de UCS para sondear los usos de suelo de carácter artificial (infraestructuras de transporte, industrial y residencial) más cercanos a dichas instalaciones.
Por último, se evalúa el empleo de SIOSE para conocer el número de hectáreas de estas instalaciones que en 2011 se situaban sobre espacios naturales protegidos.

\section{Materiales y métodos}

\section{Fuentes}

Oportunidad de la fuente SIOSE para la localización de energía solar y eólica. Para llevar a cabo los análisis y búsqueda de patrones de localización con referencias a la ocupación de suelo de las energías renovables (solar y eólica) en la península y por CCAA, se ha utilizado una fuente de UCS (usos y cobertura de suelo) que comienza a generalizarse en España. Se trata del denominado Sistema de Información de Ocupación del Suelo en España (SIOSE), esta base de datos coordina la información que en este campo tratan las Comunidades Autónomas españolas, integrando además otras bases de datos cartográficas de uso administrativo como el Sistema de Información Catastral Nacional, o el Sistema de Información Nacional de Parcelas Agrícolas. Asimismo, SIOSE también se procesa para integrarse con la base de datos CORINE Land Cover, que trabajando a una menor escala de detalle, es una fuente que tiene el objetivo de coordinar la información de usos y cobertura de suelo entre los países de la Unión Europea (UE).

SIOSE se integra dentro de las acciones impulsadas en España en el denominado Plan Nacional de Observación del Territorio (PNOT) (Villa et al., 2005), que entre sus objetivos persigue aplicar los principios de la Directiva INSPIRE (2007/2/CE) y que establece las recomendaciones para integrar la infraestructura de información espacial dentro de la UE. De este modo, SIOSE atiende a las normas generales establecidas por esta directiva, y está orientada a la aplicación de las políticas comunitarias de medio ambiente y/o a políticas o actuaciones que puedan incidir en el medio ambiente. 
La información SIOSE se construye a través de trabajos asistidos de fotointerpretación, basado en la observación de imágenes de alta resolución (SPOT5 de 2,5 m. y PNOA ${ }^{1}$ de $0,5 \mathrm{~m}$.). La unidad mínima cartográfica se encuentra entre las 2 ha, generalmente para coberturas naturales, agrícolas $\mathrm{o}$ láminas de agua, y las 0,5 ha, empleada para elementos de detalle como la vegetación de ribera o los cultivos bajo plástico. En este sentido, la distancia mínima para la captura cartográfica se establece en 15 metros, si bien para los mencionados elementos de detalle puede ser aún menor. La escala de referencia es 1:25.000 y recoge un total de 40 coberturas de tipo simple y 45 de tipo compuesto. Todas estas características permiten realizar estudios de mayor detalle sobre las coberturas y usos de suelo, a escalas donde la fuente europea CORINE Land Cover puede resultar insuficiente (Díaz-Pacheco \& Gutiérrez, 2014; Tena, 2011).

La principal característica que ha llevado al uso de SIOSE para esta investigación es que, entre las coberturas que gestiona esta base de datos, se encuentran varias referidas a las energías, y más concretamente, a la localización de energías renovables, solar y eólica. Estas coberturas se encuentran clasificadas dentro de lo que SIOSE denomina 'coberturas compuestas' y están formadas por varias coberturas asociadas que pueden ser simples o también compuestas. Esto ocurre porque cuando existen usos de suelo o coberturas diferenciadas dentro de una unidad espacial cuya superficie es menor que la unidad mínima cartográfica (hasta un detalle máximo de 0,5 ha), las coberturas $o$ usos se definen en la base de datos de acuerdo al porcentaje ocupado, aunque no tengan correspondencia con la totalidad de una de estas unidades espaciales (polígonos). Por lo tanto, SIOSE da cuenta de, al menos, aquellas instalaciones dedicadas a la energía solar y eólica.
Necesidad de uso de CORINE Land Cover para analizar los cambios de UCS. Vistas las ventajas que presenta la fuente SIOSE en cuanto a escala de detalle y a la localización de coberturas que especifican usos de suelo dedicados a energía solar y eólica, el método de almacenamiento de información de esta base de datos, presenta algunos inconvenientes para este estudio. En este sentido, cuando se quieren analizar los cambios de UCS dentro de un marco espacial explícito, las mencionadas coberturas compuestas y asociadas no llegan a aportar información de la localización y límites específicos de las zonas ocupadas por las instalaciones de energías solar y eólica.

Esta situación no ocurre del mismo modo para todos los tipos de coberturas donde aparecen las energías solar y eólica. Como se aprecia en el ejemplo de la figura 1, a través de los polígonos que representan coberturas asociadas o mosaicos irregulares (figura $1, \mathrm{~b}$ y c), no es posible concretar dónde se encuentran y cuáles son los límites que ocupan las instalaciones de energía solar dentro de este polígono (código NSL). En cambio, cuando la energía solar es el uso principal de la cobertura compuesta (figura 1, a), si bien existen diferenciaciones de uso dentro de las instalaciones, puede afirmarse que, de modo general, el polígono capturado está representando una superficie ocupada por instalaciones de energía solar.

Esta característica espacial de la fuente SIOSE se convierte en un inconveniente a la hora de tratar de emplearla para realizar análisis espaciales de cambio de UCS. Cuando las instalaciones de energía solar y eólica se identifican dentro de un polígono que representa una cobertura compuesta de asociación o de mosaico regular o irregular, no puede confirmarse cuál es la ocupación y la localización aproximada de las mencionadas instalaciones. De este modo, a excepción de aquellos polígonos donde las instalaciones de energía solar y eólica representan la

1 PNOA: Plan Nacional de Ortofotografía Aérea 
cobertura principal (figura 1, a), para el resto de coberturas compuestas y asociadas donde se representan estas instalaciones, utilizando SIOSE no pueden realizarse aproximaciones espaciales explícitas sobre la cobertura de suelo que han ocupado, respecto a un periodo temporal anterior. Este hecho se ilustra en la figura 2, donde se muestra como un polígono que representa instalaciones de energía solar en 2011, ha ocupado un polígono (figura 2, polígono 2) que representa multitud de coberturas que no tienen una correspondencia espacial explícita, más allá de encontrarse dentro del mencionado polígono.

Para intentar suplir este inconveniente, y para alcanzar los objetivos planteados en el presente trabajo, se ha acudido a la fuente CORINE Land Cover (CLC) de uso común y extendido dentro de la Unión Europea, que trabaja a una escala cartográfica de menor detalle respecto a SIOSE (1:200.000), sin embargo ésta resulta de utilidad en este estudio, dado que las clases y/o coberturas que representa tienen siempre una correspondencia espacial explícita. De este modo, tal y como se observa en el ejemplo de la figura 3, superponiendo los polígonos SIOSE, 2011, de cobertura principal

a

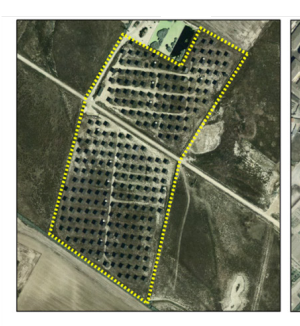

$200 \mathrm{~m}$ energía solar con los polígonos "simples" de CLC, 2000, se obtiene el resultado de las instalaciones energéticas observadas en 2011 (SIOSE), que han ocupado un espacio dedicado a frutales en el año 2000 (CLC).

En este trabajo se combinan por tanto el uso de dos fuentes de información de UCS, que si bien no operan a la misma escala cartográfica, aportan, en el caso de SIOSE, una ingente información sobre usos de suelo; entre los que nos interesan para este estudio aquellas coberturas que identifican las instalaciones de energía solar y eólica. Por otro lado, CORINE Land Cover, si bien no contiene datos referentes a estos usos, funciona muy bien como soporte de coberturas simples. Estas coberturas, aunque es cierto que son menos precisas en términos de escala aportan, sin embargo, localizaciones espaciales explícitas para los cambios de UCS relativos a instalaciones de energía solar y eólica.

En la tabla 1 se muestran las coberturas y las clases de SIOSE y CLC que han sido empleadas en los análisis de información espacial llevados a cabo en este trabajo, recogidas en el siguiente apartado metodológico.

b

c
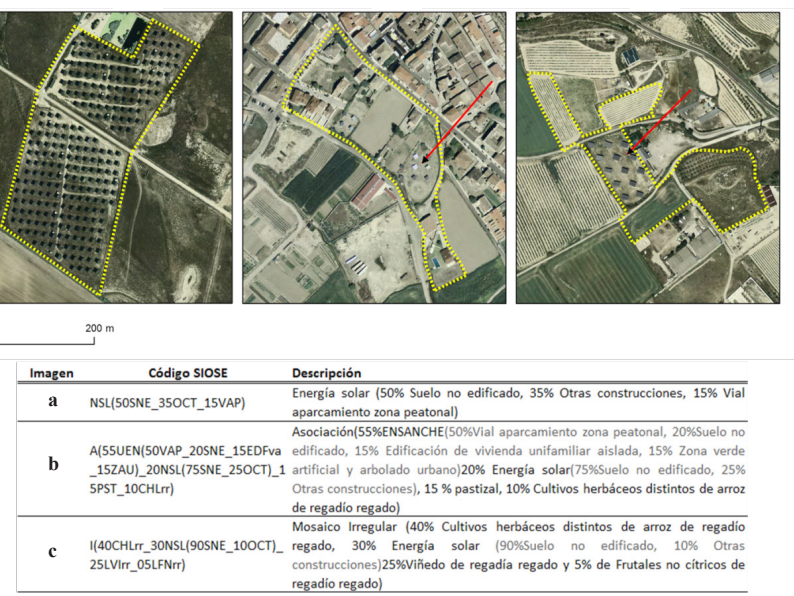

Figura 1. Polígonos, códigos SIOSE y localización de instalaciones de energía solar. Fuentes: SIOSE, 2011; PNOA, 2017.

Figure 1. Polygons, SIOSE codes and locations of solar power farms. Source: SIOSE, 2011; PNOA, 2017. 


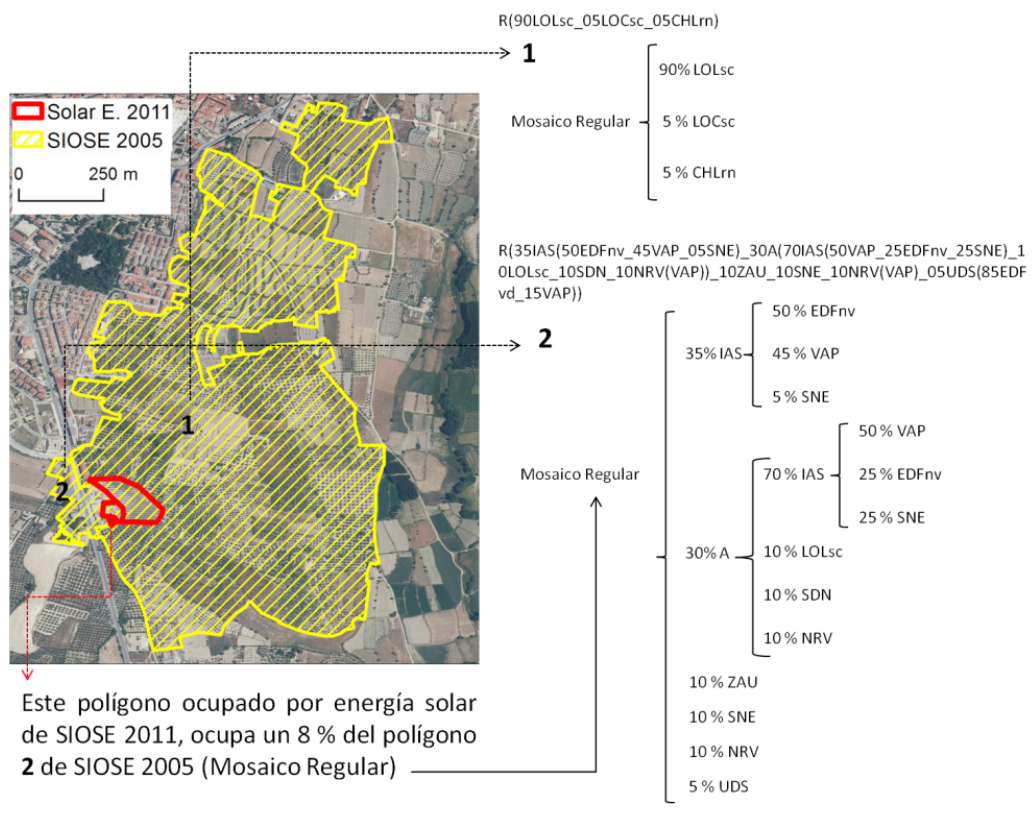

Figura 2. Ejemplo de polígonos SIOSE, 2011 representando instalaciones de energía solar, sobre polígonos SIOSE, 2005 representando coberturas compuestas. Fuentes: SIOSE, 2011, PNOA, 2017.

Figure 2. Example of SIOSE, 2011 polygon representing a solar power farm, over a SIOSE, 2005 polygon representing composed coveranges. Source: SIOSE, 2011, PNOA, 2017.

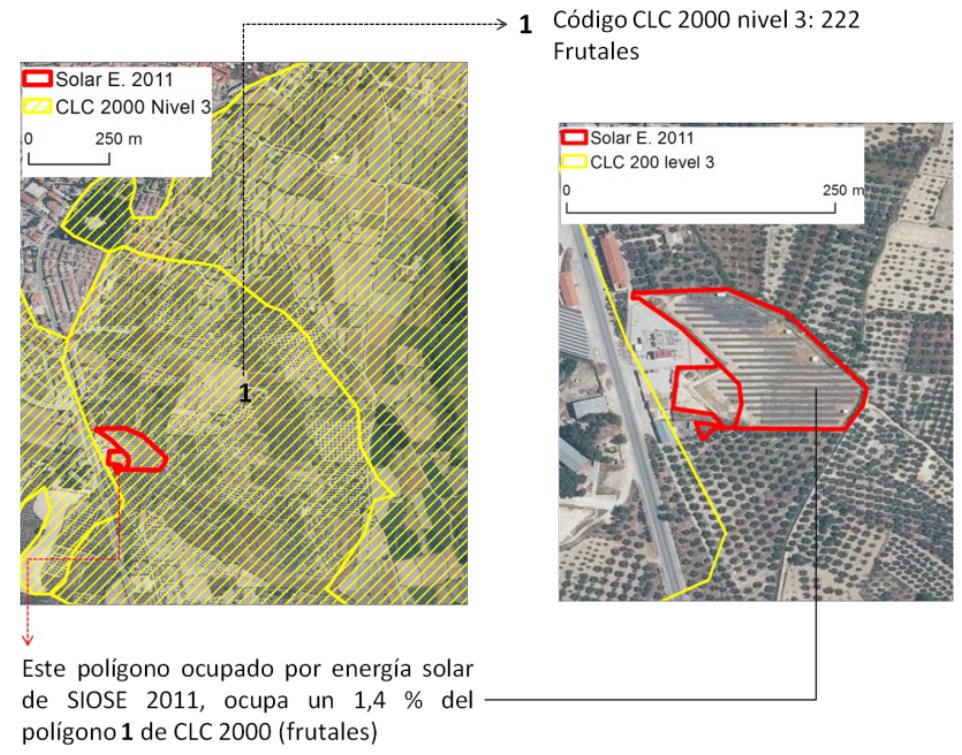

Figura 3. Ejemplo de polígonos SIOSE, 2011 representando instalaciones de energía solar, sobre polígonos CORINE 2000 representando coberturas simples. Fuentes: SIOSE, 2011, CLC, 2006, PNOA, 2017.

Figure 3. Example of SIOSE, 2011 polygon, representing a solar power farm over CLC, 2000 polygons, representing simple coveranges. Source: SIOSE, 2011, CLC, 2006, PNOA, 2017. 


\section{Tabla 1}

UCS energía en SIOSE y UCS en CORINE Land Cover, Nivel 3. Fuente: CLC, 2006.

Table 1

LULC power on SIOSE and LULC on Level 3 of CORINE Land Cover. Source: CLC, 2006.

\begin{tabular}{|c|c|c|c|c|c|}
\hline \multicolumn{2}{|c|}{$\begin{array}{l}\text { UCS SIOSE (Energías) 3.5 Artificial Compuesto/3.5.6 } \\
\text { Infraestructuras/3562 Energía }\end{array}$} & \multicolumn{3}{|c|}{ UCS CORINE Land Cover, Nivel 3} & \multirow[b]{2}{*}{ Bosques de frondosas } \\
\hline 35621 & EÓLICA (NEO) & 111 & Tejido urbano continuo & 311 & \\
\hline $\begin{array}{l}\text { Tipo 1: Eólica es uso } \\
\text { principal }\end{array}$ & $\begin{array}{l}\text { Código SIOSE: } \\
\operatorname{NEO}(\ldots)\end{array}$ & 112 & Tejido urbano discontinuo & 312 & Bosques de coníferas \\
\hline $\begin{array}{l}\text { Tipo 2: Eólica es uso } \\
\text { asociado }\end{array}$ & $\begin{array}{l}\text { Código SIOSE: } \\
\text { A( ...NEO ...),R( ...NEO ...), } \\
\text { I( ...NEO ...) }\end{array}$ & 121 & $\begin{array}{l}\text { Zonas industriales o } \\
\text { comerciales }\end{array}$ & 313 & Bosque mixto \\
\hline 35621 & SOLAR (NSL) & 122 & $\begin{array}{l}\text { Redes viarias, ferroviarias y } \\
\text { terrenos asociados }\end{array}$ & 321 & Pastizales Naturales \\
\hline $\begin{array}{l}\text { Tipo 1: Solar es uso } \\
\text { principal }\end{array}$ & $\begin{array}{l}\text { Código SIOSE: } \\
\text { NSL(...) }\end{array}$ & 123 & Zonas portuarias & 322 & $\begin{array}{l}\text { Landas y matorrales } \\
\text { mesófilos }\end{array}$ \\
\hline \multirow[t]{17}{*}{$\begin{array}{l}\text { Tipo 2: Solar es uso } \\
\text { asociado }\end{array}$} & $\begin{array}{l}\text { Código SIOSE: } \\
\text { A( ...NSL ...),R( ...NSL ...), } \\
\text { I( ...NSL ...) }\end{array}$ & 124 & Aeropuertos & 323 & Matorrales esclerófilos \\
\hline & & 131 & Zonas de extracción minera & 324 & $\begin{array}{l}\text { Matorral boscoso de } \\
\text { transición }\end{array}$ \\
\hline & & 132 & Escombreras y vertederos & 331 & Playas, dunas y arenales \\
\hline & & 133 & Zonas en construcción & 332 & Roquedo \\
\hline & & 141 & Zonas verdes urbanas & 333 & $\begin{array}{l}\text { Espacios con vegetación } \\
\text { escasa }\end{array}$ \\
\hline & & 142 & $\begin{array}{l}\text { Instalaciones deportivas y } \\
\text { recreativas }\end{array}$ & 334 & Zonas quemadas \\
\hline & & 211 & Tierras de labor en secano & 335 & $\begin{array}{l}\text { Glaciares y nieve } \\
\text { permanente }\end{array}$ \\
\hline & & 212 & $\begin{array}{l}\text { Terrenos regados } \\
\text { permanentemente }\end{array}$ & 411 & $\begin{array}{l}\text { Humedales y zonas } \\
\text { pantanosas }\end{array}$ \\
\hline & & 213 & Arrozales & 412 & Tuberías y prados turbosos \\
\hline & & 221 & Viñedos & 421 & Marismas \\
\hline & & 222 & Frutales & 422 & Salinas \\
\hline & & 223 & Olivares & 423 & Zonas llanas intermareales \\
\hline & & 231 & Prados y praderas & 511 & Cursos de agua \\
\hline & & 241 & $\begin{array}{l}\text { Cultivos anuales asociados } \\
\text { con cultivos }\end{array}$ & 512 & Láminas de agua \\
\hline & & 242 & Mosaico de cultivos & 521 & Lagunas costeras \\
\hline & & 243 & $\begin{array}{l}\text { Terrenos principalmente } \\
\text { agrícolas, pero con } \\
\text { importantes espacios } \\
\text { de vegetación natural y } \\
\text { seminatural }\end{array}$ & 522 & Estuarios \\
\hline & & 244 & Sistemas agroforestales & 523 & Mares y océanos \\
\hline
\end{tabular}


Se indica en esta sección sobre fuentes, el empleo de información del proyecto Shuttle Radar Topography Mission de la Administración Nacional de la Aeronáutica y del Espacio de Estados Unidos (NASA) para la obtención de los datos de altitud proporcionados por el modelo digital de elevaciones desarrollado en este proyecto. Por último, la información geográfica de los Espacios Naturales Protegidos, provista por el Ministerio de Agricultura, Alimentación y Medio Ambiente de España, creados en 1994 y con una actualización en 2016.

\section{Método}

Para alcanzar los objetivos y resolver las cuestiones que se plantean en este estudio se han realizado una serie de ejercicios de valoración sobre las fuentes de información SIOSE y CLC que se señalan a continuación.

Valoración 1: Análisis de distribución espacial de las instalaciones energéticas solar y eólica registradas en SIOSE 2005 y 2011.

Valoración 2: Análisis de patrones de ocupación de UCS relativos a las instalaciones energéticas solar y eólica a través de SIOSE 2011 y CLC 2006.

Valoración 3: Análisis de patrones de localización de las instalaciones energéticas solar y eólica respecto a la altitud (SIOSE 2011).

Valoración 4: Análisis de localización de las instalaciones de energía solar y eólica respecto a las estructuras urbanas más cercanas, a través de CLC 2006 y SIOSE 2011.

Valoración 5: Estudio del emplazamiento de las instalaciones de energía solar y eólica sobre espacios naturales protegidos.

Valoración 1: Análisis de distribución espacial de las instalaciones energéticas solar y eólica registradas en SIOSE 2005 y 2011. Se elaboró un análisis cuantitativo y exploratorio basado, principalmente, en SIOSE, dónde se realizaron una serie de consultas a la base de datos, estando éstas, a su vez, relacionadas con los límites de las Comunidades Autónomas (CCAA) españolas dentro de la Península Ibérica. A través de estas consultas se han conocido, de manera aproximada, las hectáreas de suelo que cada CCAA dedica a acoger instalaciones de energía eólica y solar, en referencia a los periodos de SIOSE, es decir 2005 y 2011. En este sentido, se obtuvieron también datos y representaciones gráficas de la evolución de la ocupación de suelo en la Península Ibérica y por CCAA, en lo que se refiere a instalaciones de energía solar y eólica.

Para realizar el recuento de ha dedicadas a energía solar y eólica dentro de los polígonos compuestos de SIOSE, se lleva a cabo un cálculo a través del porcentaje indicado en la base de datos. De este modo, si un polígono tiene 120 ha y se indica un $10 \%$ de energía solar (10NSL), se computan 12 ha de suelo dedicado a la producción de energía solar.

Valoración 2: Elaboración de un ranking de ocupación de UCS relativos a las instalaciones energéticas solar y eólica a través de SIOSE 2011 y CLC 2006. Se lleva a cabo un análisis espacial, sobre la evolución de la ocupación del suelo por instalaciones de energía solar y eólica, aunque en este caso orientado a conocer qué otros usos o cobertura de suelo están ocupando este tipo de instalaciones. En este caso la exploración se realiza entre el periodo 2006 (CLC) y 2011 (SIOSE). Los motivos para la combinación de estas bases de datos en el análisis han sido ya explicados en el apartado anterior (fuentes). En este análisis, las clases de usos de suelo para CLC Nivel 3 fueron reclasificadas en un número inferior de categorías con el fin de simplificar el análisis (tabla 2).

Valoración 3: Análisis de patrones de localización de las instalaciones 
energéticas solar y eólica respecto a la altitud y los datos de SIOSE 2011. Utilizando los datos de altitud en formato raster suministrados por la misión de la NASA, SRTM (Shuttle Radar Topography Mission) (Rabus, Eineder, Roth, \& Bamler, 2003). Se computaron a través de un SIG, las hectáreas correspondientes a las instalaciones de energía solar y eólica de acuerdo a su altitud, con el objetivo de detectar patrones de distribución general respecto a esta variable.

Valoración 4: Análisis de localización de las instalaciones de energía solar y eólica respecto a las estructuras urbanas más cercanas, a través de CLC 2006 y SIOSE 2011. Empleando CORINE Land Cover (2006) y SIOSE (2011), se exploran a través de un SIG, los usos de suelo artificial (infraestructuras de transporte, industrial-comercial y residencial) que se encuentran a menor distancia euclidiana de las instalaciones de energía solar y eólica.

Valoración 5: Estudio del emplazamiento de las instalaciones de energía solar y eólica sobre espacios naturales protegidos. Se contabilizan las hectáreas de las diferentes instalaciones de generación de energía solar y eólica (SIOSE 2011) que se ubican sobre espacios naturales protegidos, de acuerdo a la información registrada en la base de datos del Ministerio de Agricultura, Alimentación y Medio Ambiente del Gobierno de España.

\section{Área de estudio}

La exploración de SIOSE como fuente para el análisis de la implantación territorial de la producción de energía eólica y solar, conlleva, de manera inevitable, a centrar los análisis en España, dado que esta base

\section{Tabla 2}

Nombre asignado a usos de suelo CLC Nivel 3, para simplificar el análisis. Fuente: CLC, 2006.

Table 2

Land use categories classification on CLC at level 3 to simplify the analysis. Source: CLC, 2006.

\begin{tabular}{|c|c|c|c|c|c|}
\hline CODE CLC & $\begin{array}{l}\text { Nombre } \\
\text { análisis }\end{array}$ & CODE CLC & $\begin{array}{l}\text { Nombre } \\
\text { análisis }\end{array}$ & CODE CLC & $\begin{array}{l}\text { Nombre } \\
\text { análisis }\end{array}$ \\
\hline 111 y 112 & Urbano & 211 & Secano & 311,312 y 313 & Bosque \\
\hline 121 & Industrial & 212 у 213 & Regadío & 321 & Prado \\
\hline 122 & Carretera & 221 & Viñedo & 322,323 у 324 & Matorral \\
\hline 123 y 124 & Puerto & 222 & Frutal & $\begin{array}{l}331,332,333, \\
412,421,422 \\
\text { у } 423\end{array}$ & Natural \\
\hline 131 & Minera & 223 & Olivar & 334 & Quemado \\
\hline 132 у 133 & Degradado & 231 & Prado & 335 & Montaña \\
\hline \multirow[t]{3}{*}{141 y 142} & Ocio & 241,242 у 243 & Cultivo & 411 & Humedal \\
\hline & & \multirow[t]{2}{*}{244} & \multirow[t]{2}{*}{ Agronatural } & $\begin{array}{l}511,512,521 \\
\text { у } 522\end{array}$ & Agua \\
\hline & & & & 523 & Mar \\
\hline
\end{tabular}


de datos en concreto, se ha desarrollado de manera individual en este país. No obstante, debido a que en este trabajo se combina el empleo de CLC, de uso extendido en Europa, la metodología podría ser extrapolable a otras áreas de estudio donde existen bases de datos geográficas con coberturas referentes a usos de suelo dedicados a producir energía eólica y solar.

Si bien la base de datos SIOSE engloba todo el territorio español, continental e insular, este estudio se ha enfocado en el espacio continuo del territorio continental español (peninsular), considerando que tanto en las Islas Canarias como en las Islas Baleares, pueden darse otro tipo de patrones en función de su estructura geográfica insular con un territorio fragmentado de reducidas dimensiones y con una compleja topografía.

España, desde 1990 el desarrollo de la energía renovable solar y eólica ha tenido un crecimiento espectacular hasta 2003 (Alonso et al., 2016) y, aunque a menor ritmo, la tendencia se ha mantenido durante la primera década del siglo XXI, donde se engloba el periodo de estudio de este trabajo. Los motivos de la exitosa implantación de las energías renovables (EERR) en España, se deben a un cúmulo de factores, varios autores señalan la importancia de las tarifas especiales de producción aplicadas a las renovables (feed-in tariff), un sistema de subvención que garantizaba a largo plazo la compra de electricidad procedente de fuentes renovables a un precio atractivo (Del Río \& Gual 2007, Del Río 2008, Couture \& Gagnon, 2010).

Por el contrario, autores como Dinica (2008) sugiere que la explicación al desarrollo de EERR se debe fundamentalmente al apoyo por parte de la administración desde la década de los 80, concretamente a los acuerdos específicos entre las empresas privadas y el sector público, los llamados partenariados público privados. Estas explicaciones, no obstante, suelen obviar las marcadas diferencias entre CCAA respecto a la implantación y desarrollo de las EERR, estas variaciones no responden únicamente a causas o condicionantes naturales. Alonso et al. (2016), a raíz de una investigación sobre el papel de los diversos actores en el sector de las EERR, demuestra clara diferencias entre el carácter y fortaleza de los vínculos entre la ciudadanía, el sector IDi, las administraciones regionales y el sector privado en las distintas CCAA españolas. Estas diferencias podrían explicar, al menos en parte, el contrastado patrón de desarrollo entre, por un lado, CCAA como Extremadura, donde la energía eólica no ha llegado, y por otro, Navarra, región puntera y pionera en las EERR en España (Faulín, Lera, Pintor, \& García, 2006, Hewitt, Winder, Jiménez, Alonso, \& Bermejo, 2017).

También, destaca, como resultado de este trabajo, la diferencia entre los patrones de desarrollo de los dos tipos de energía investigado. La anteriormente comentada Extremadura, que no cuenta con instalaciones de energía eólica, experimentó una fuerte expansión de energía solar entre 2005 y 2011. Es notable, que las CCAA con más hectáreas de energía solar, que son, con diferencia, Andalucía, Castilla la Mancha y Extremadura también contaban con grandes bolsas de suelo de cultivo o prado de escasa rentabilidad. Así que, cuando las instituciones públicas fomentaron la expansión de las granjas solares a mediados de la primera década del milenio, proceso que tuvo éxito más allá de las expectativas, dando lugar a cambios de política para calmar el "boom solar" (del Río \& Mir-Artigues, 2012), se facilitó la transformación de grandes extensiones de escaso uso agrícola en estas comunidades en instalaciones solares.

Todo ello unido a que España cuenta con numerosos emplazamientos idóneos para la implantación, tanto de energía eólica como solar. Debido a las extensas áreas montañosas y costeras, el territorio peninsular presenta unas condiciones muy proclives a la implantación de energía eólica (Ignateva \& Pérez, 2008). El litoral y los 
sistemas montañosos registran los vientos más intensos y constantes (Aymamí, 2011) en una gran cantidad de sectores (Olcina Cantos, 1994), tanto vientos regionales como locales de gran entidad determinados, en buena medida, por la distribución de las unidades de relieve (Martín Vide \& Olcina Cantos, 2001) y las formas de la costa.

También, la energía solar en la España peninsular, cuenta con unas excepcionales condiciones climáticas con unos registros de insolación que en casi toda la península superan la isohelia de las 2.000 horas, umbral a partir del cual los usos de la energía solar pueden cubrir parte de las necesidades domésticas (Castro, Martín-Vide, \& Alonso, 2005). En España llegan a alcanzarse las 3.000 horas en los sectores más soleados de la Costa de la Luz entre Cádiz y Huelva (Martín Vide \& Olcina Cantos, 2001) y rozando ese valor en sectores del Sureste peninsular según indican los datos de la red de estaciones meteorológicas de AEMET.

\section{Resultados}

\section{Valoración sobre el análisis de distribución espacial de las hectáreas de instalaciones}

\section{energéticas solar y eólica registradas en SIOSE 2005 y 2011}

De acuerdo a la fuente utilizada, la primera información relevante que se extrae de este análisis es el incremento indiscutible de las hectáreas ocupadas por instalaciones de energía renovable solar y eólica en España, entre los años 2005 y 2011, tal y como ha ocurrido globalmente en el planeta (Grupo Intergubernamental de Expertos sobre el Cambio Climático, IPCC, 2011). A partir de la Base de Datos (bbdd) SIOSE, se estima que la energía solar ocupa 22.009,66 ha en 2011 a partir de las 689,84 ha que se registraron en 2005 , lo que se traduce en un incremento de un 3.090,5\%. Del mismo modo, aunque en menor proporción, las hectáreas ocupadas por la energía eólica pasan de 7.283,31 ha en 2005, a 12.681,15 ha en 2011, con un crecimiento porcentual del 74,1\% (figura 4).

En el análisis por Comunidades Autónomas los incrementos siguen un patrón general al observado en el territorio español peninsular, si bien existen importantes diferencias en el desarrollo de la ocupación de suelo por este tipo de instalaciones de energía en las distintas
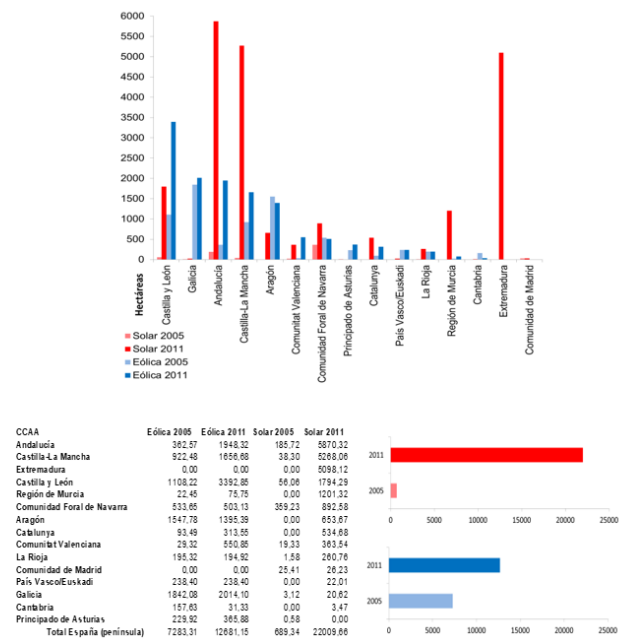

Figura 4. Energía solar y eólica en España (península) y por CCAA 2005-2011. Fuente: SIOSE, $2005,2011$.

Figure 4. Solar and wind power in Spain (peninsula) and by Autonomous Community 2005-2011. Source: SIOSE, 2005, 2011. 
regiones. Andalucía, Castilla-La Mancha, Extremadura y Castilla León muestran los mayores incrementos en superficie dedicada a energía solar, mientras que los parques eólicos crecen más y ocupan más hectáreas de suelo en las regiones de Castilla y León, Galicia, Andalucía y Aragón (figura 4).

Realizando un análisis espacial sobre la densidad de hectáreas de instalaciones solares y eólica en la península se observa un crecimiento exponencial de ambas energías, si bien en el caso de la energía eólica se obsvverva aún en 2011 un vacío hacia el centro y el oeste peninsular, definido por Extremadura y el Norte de Andalucía. En la densidad de hectáreas de instalaciones de energía solar, sorprende el caso de La Rioja, que en 2005 destaca de manera muy significativa sobre el resto del Territorio español peninsular (figura 5).

No existe una fuente de información en España que permita validar el incremento de las hectáreas de suelo ocupado por parques solares y eólicos obtenido a través de SIOSE entre 2005 y 2011 . En términos de producción energética, para la solar fotovoltaica, que es la que generalmente se instala en los parques solares, se pasó de una producción en 2005 inferior a $100 \mathrm{GWh}$ a producir $7.200 \mathrm{GWh}$ en 2011 (Red Eléctrica de España (REE), 2017). Si bien el incremento relativo no es proporcional a la ocupación de hectáreas que arroja el SIOSE, sí se confirma el crecimiento exacerbado de la producción solar durante el periodo estudiado. Para el caso de la energía eólica el incremento de producción entre 2005-2011 fue de 21.000 GWh, duplicando en 2011 la producción de 2005 (REE, 2017). El patrón de distribución de hectáreas de parques eólicos y solares en España obtenido a través de SIOSE para 2011 (figura 5) guarda similitud con el patrón que indica la estadística actual de Red Eléctrica de España, para la distribución del número de instalaciones en 2016 (figura 6) (REE, 2017).

En el análisis por Comunidades Autónomas los incrementos siguen un patrón general al observado en el territorio español peninsular, si bien existen importantes diferencias en el desarrollo de la ocupación de suelo por este tipo de instalaciones de energía en las distintas regiones. Andalucía, CastillaLa Mancha, Extremadura y Castilla León muestran los mayores incrementos en superficie dedicada a energía solar, mientras que los parques eólicos crecen más y ocupan más hectáreas de suelo en las regiones de Castilla y León, Galicia, Andalucía y Aragón (figura 4).

Realizando un análisis espacial sobre la densidad de hectáreas de instalaciones solares y eólica en la península se observa un crecimiento exponencial de ambas energías, si bien en el caso de la energía eólica se observa aún en 2011 un vacío hacia el centro y el oeste peninsular, definido por Extremadura y el Norte de Andalucía. En la densidad de hectáreas de instalaciones de energía solar, sorprende el caso de La Rioja, que en 2005 destaca de manera muy significativa sobre el resto del Territorio español peninsular (figura 5).

No existe una fuente de información en España que permita validar el incremento de las hectáreas de suelo ocupado por parques solares y eólicos obtenido a través de SIOSE entre 2005 y 2011. En términos de producción energética, para la solar fotovoltaica, que es la que generalmente se instala en los parques solares, se pasó de una producción en 2005 inferior a $100 \mathrm{GWh}$ a producir $7.200 \mathrm{GWh}$ en 2011 (REE, 2017). Si bien el incremento relativo no es proporcional a la ocupación de hectáreas que arroja el SIOSE, si se confirma el crecimiento exacerbado de la producción solar durante el periodo estudiado. Para el caso de la energía eólica el incremento de producción entre 2005-2011 fue de 21.000 GWh, duplicando en 2011 la producción de 2005 (REE, 2017).

El patrón de distribución de hectáreas de parques eólicos y solares en España obtenido a través de SIOSE para 2011 (figura 5) guarda similitud con el patrón que indica 


\section{Energía solar 2005}

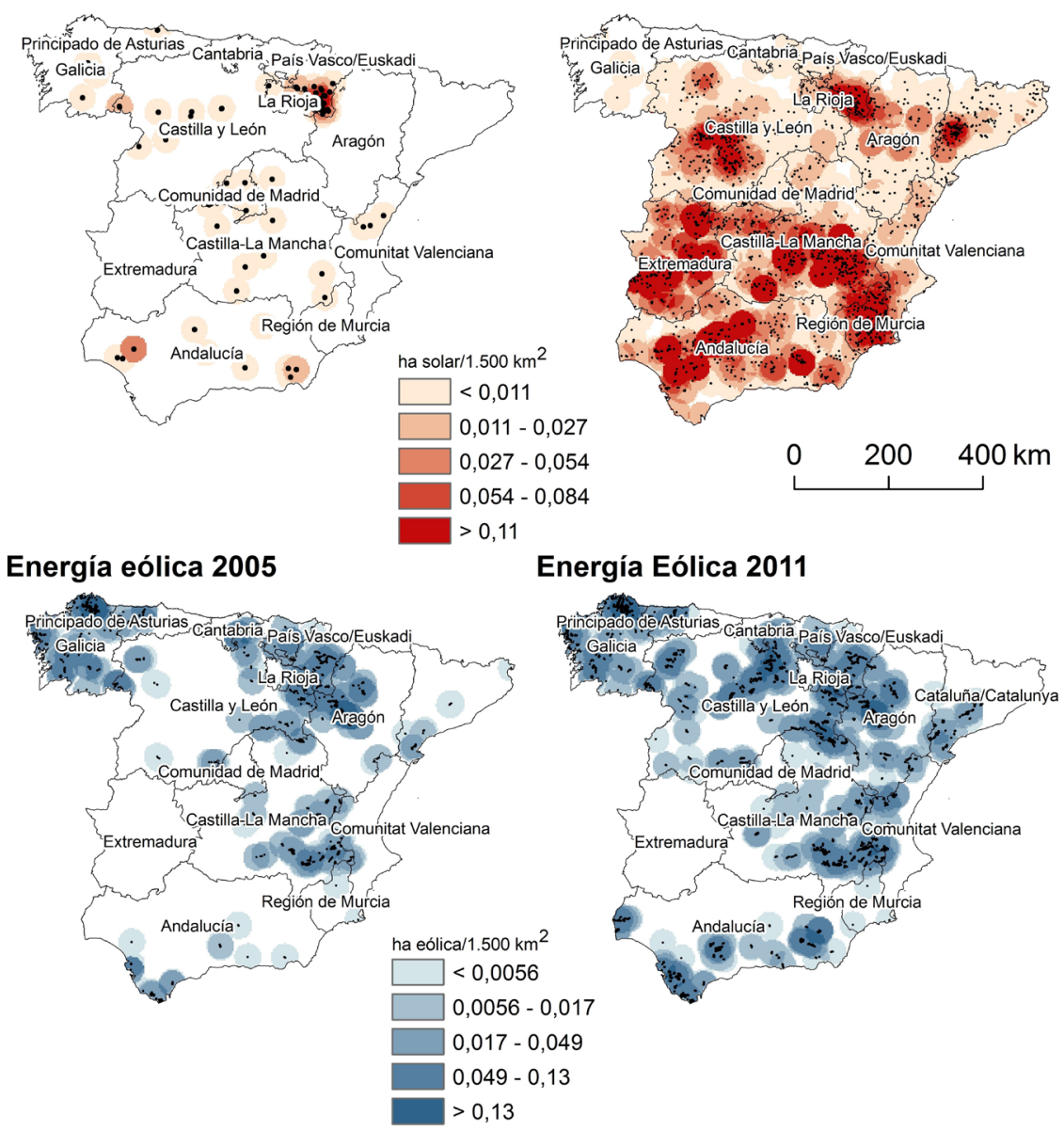

Figura 5. Densidad de hectáreas solar y eólica en 2005 y 2011. Fuente: SIOSE, 2005, 2011.

Figure 5. Spain (penisula) Density of solar and wind power cover. Source: SIOSE, 2005, 2011.

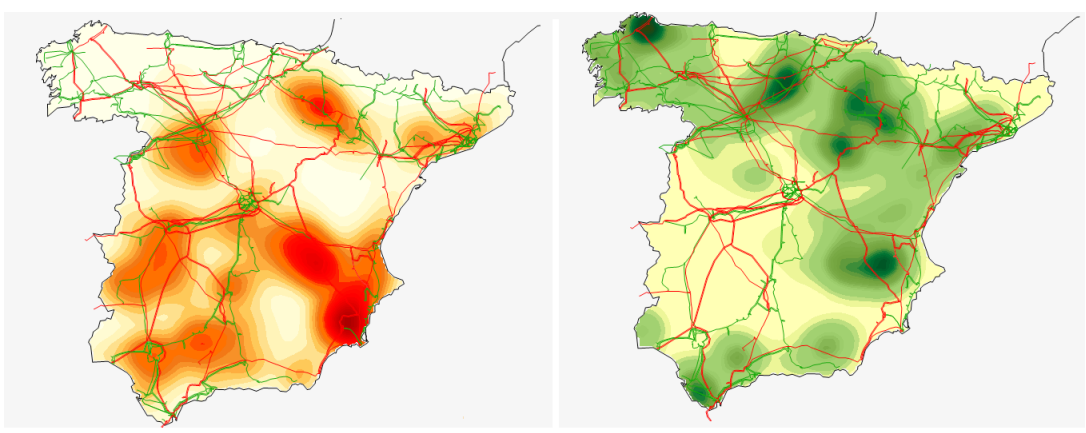

Figura 6. Distribución de las instalaciones de energía solar (izquierda) y eólica (derecha) en España penínsular en 2016. Fuente: REE, 2017.

Figure 6. Solar and wind power farms distribution on Spain (peninsula) in 2016. Source: REE, 2017. 
la estadística actual de Red Eléctrica de España, para la distribución del número de instalaciones en 2016 (figura 6) (REE, 2017).

Valoración sobre la elaboración de un ranking de ocupación de UCS relativos a las instalaciones energéticas solar y eólica a través de SIOSE, 2011 y CLC, 2006

Energía eólica. Cuando se superponen los datos de SIOSE (2011) sobre los UCS de CLC (2006) delimitados en la base de datos CORINE Land Cover se observa como las instalaciones de energía eólica suelen emplazarse, principalmente, sobre coberturas que generalmente no obedecen a usos antrópicos de profunda transformación, como superficies cubiertas por matorral, bosque, prado y espacios a los que CLC denomina agronaturales. En esta categoría de suelo existe algún tipo de aprovechamiento agrícola o forestal, pero no se observan cultivos extensos y claramente definidos. Posteriormente los suelos dedicados al cultivo de secano y otros tipos mixtos no irrigados mantienen en todas las CCAA una posición media en el ranking de suelos empleados para la instalación de campos eólicos y, por último, suelos naturales (muy posiblemente roquedos y estepas) y viñedos, aparecen también alrededor del décimo lugar en un ranking de 16 categorías. En algunas regiones como en Murcia tiene importancia la ocupación de suelo dedicado a la actividad minera, o en otras como en Castilla León y Galicia, los suelos quemados alcanzan alguna posición entre los 6 primeros. Andalucía y Castilla-La Mancha, comunidades donde las instalaciones eólicas muestran un desarrollo más contundente, tienen un número considerable de hectáreas sobre suelo de regadío y frutal. Por último, destacar el caso de Andalucía donde un porcentaje considerable de superficie con instalaciones eólicas se encuentra sobre suelo urbano o suelo degradado (tabla 3 y figura 7).

Energía solar. En el caso de la energía solar el ranking de UCS ocupados por estas instalaciones se disputa entre 19 de los tipos clasificados (tabla 4 y figura 8). De manera general, los UCS principalmente ocupados son aquellos clasificados como cultivos de secano, seguidos por cultivos en general (no irrigados), superficies agronaturales y posteriormente áreas de matorral, viñedo, bosque y prado. En Andalucía tienen cierta posición (entre los 6 primeros) el olivar y los suelos naturales, así como en Murcia destaca la ocupación de áreas de frutal en contraposición con otras CCAA. En el caso de la solar, el suelo urbano e industrial se encuentran entre el décimo y el duodécimo lugar en regiones como Andalucía, CastillaLa Mancha, Catilla León, Catalunya y comunidad Valenciana donde, por otro lado, existen más de 500 hectáreas de energía solar instaladas en todas ellas.

El uso de SIOSE y CLC permite por tanto en este caso identificar geográficamente y cuantificar el tipo de suelo que se está ocupando con energías renovables. En esta valoración se ha empleado un ranking simple comparativo, pero además de la información obtenida, queda evidencia de la utilidad y el valor que aporta el uso complementario de ambas bbdd. Actualmente no se han identificado trabajos o fuentes que permitan valorar comparativamente los resultados expuestos para el área de estudio.

Valoración sobre el análisis de patrones de localización de las instalaciones energéticas solar y eólica respecto a la altitud a través de SIOSE 2011

A partir de este análisis se desprende, sobre todo, una diferencia clara entre la localización altitudinal de las instalaciones de energía eólica y aquellas dedicadas a la producción de energía solar. Más de un 80 $\%$ de las últimas se sitúan por debajo de los 750 m.s.n.m. Mientras que por debajo de los 250 m.s.n.m. se emplazan casi un 30\% de las mismas (figura 9).

Para el caso de la energía eólica, casi un $80 \%$ de superficie instalada se sitúa entre los 250 m.s.n.m. y los 1.250 m.s.n.m. Además, 


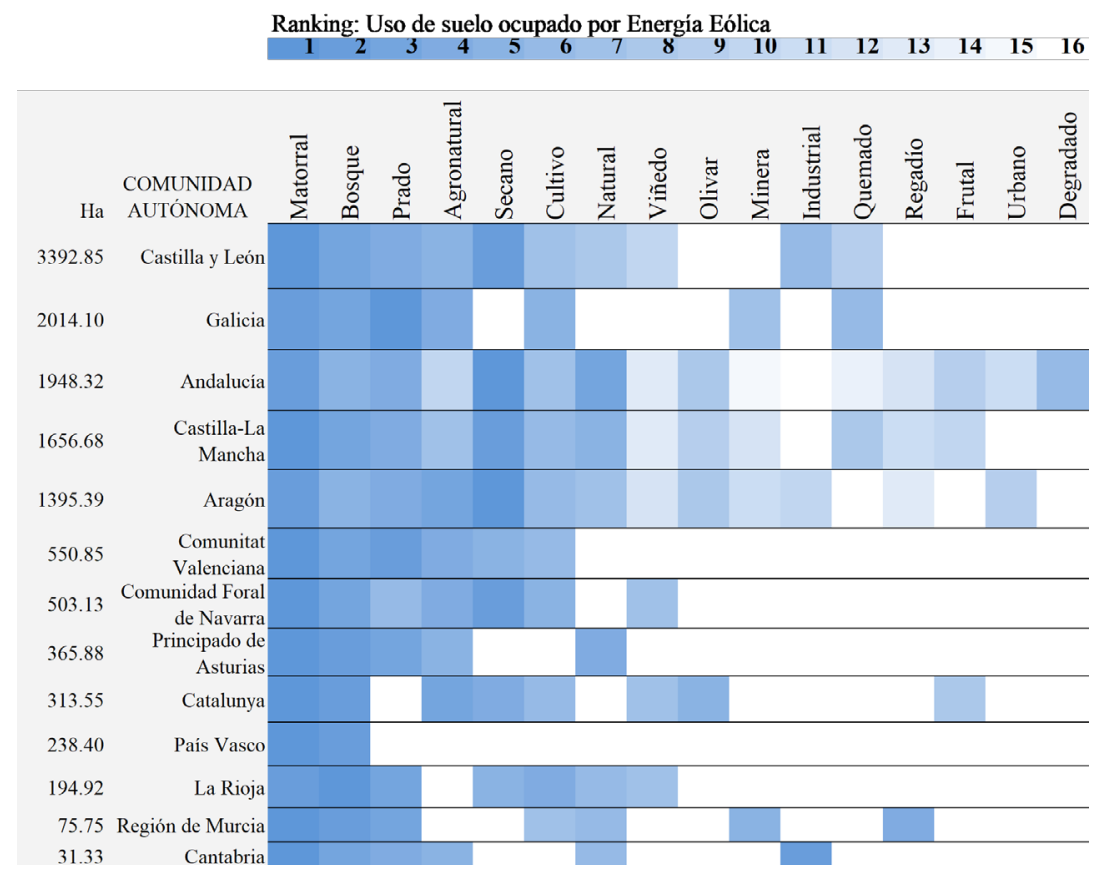

Figura 7. Usos de suelo (2006) ocupados por energía eólica 2011). Fuente: CLC, 2006; SIOSE, 2011.

Figure 7. Spain (penisula) Density of solar and wind power cover. Source: SIOSE 2005, 2011.

por encima de los 750 m.s.n.m., en clara diferencia con la energía solar, encontramos más de la mitad de hectáreas dedicadas a la producción energética eólica (figura 9).

La información obtenida a través de SIOSE complementada con una variable del terreno, en este caso la altitud, no supone ningún hallazgo imprevisible, aun cuando se realiza el análisis por Comunidad Autónoma (figura 8). Además, la altitud media de algunas Comunidades Autónomas (existencia de mesetas con altas medias de altitud) hace que la variable altitud no tenga relevancia desde un punto de vista inquiridor. Se señala entonces, la capacidad de SIOSE para ser complementada con otras variables derivadas de modelos digitales del terreno, como puede ser la orientación.

Valoración sobre el análisis de localización de las instalaciones de energía solar y eólica respecto a las estructuras urbanas más cercanas, a través de CLC 2006 y SIOSE 2011
A través de este análisis se observa, de manera clara, como las zonas ocupadas por espacios residenciales son las que, en general en el territorio peninsular español, se encuentran más cerca de las instalaciones de energía solar (el 79,01\%) y energía eólica (el $76,83 \%$ ) (figura 10).

Alrededor de un $20 \%$ de las zonas de uso de suelo industrial-comercial, fueron identificadas como el área urbana más cercana a las instalaciones energéticas (19,95\% solar y $18,10 \%$ eólica) (tabla 4). En comunidades como Castilla-León o La Rioja, el porcentaje de áreas industriales que se identifican como el suelo urbano más cercano a las instalaciones energéticas eólicas llega a alcanzar el 29\%; el mismo caso en Cantabria llega a superar el $47 \%$, aunque debe tenerse en cuenta la baja implementación de estas instalaciones en esta Comunidad (35 ha, figura 6) (tabla 4).

Los aeropuertos, puertos y redes de transporte por carretera resultaron ser las áreas que en menor proporción se identifican 
Tabla 3

Superficie (en hectáreas) de usos de suelo (2006) utilizados para energía eólica (2011). Fuente: CLC, 2006; SIOSE, 2011.

Table 3

Area (in hectares) of land uses (2006) used for wind power (2011). Source: CLC, 2006; SIOSE, 2011.

\begin{tabular}{|c|c|c|c|c|c|c|c|c|c|c|c|c|c|c|c|c|c|}
\hline $\begin{array}{l}\text { Hectáreas / } \\
\text { Energía Eólica }\end{array}$ & 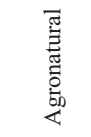 & $\begin{array}{l}\stackrel{0}{0} \\
\bar{v}_{0} \\
0 \\
0\end{array}$ & $\stackrel{\stackrel{?}{\Xi}}{\Xi}$ & 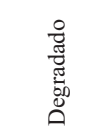 & 䙔 & 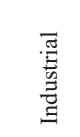 & 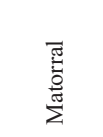 & 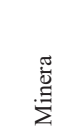 & $\begin{array}{l}\overline{\widetilde{J}} \\
\text { 壳 } \\
\text { Z }\end{array}$ & 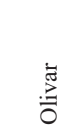 & 覂 & 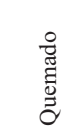 & 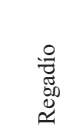 & $\begin{array}{l}\text { ̊ } \\
\text { ల్ల } \\
\text { ஸू }\end{array}$ & 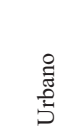 & 离 & $\begin{array}{l}\frac{\mathbb{U}}{0} \\
\frac{0}{0} \\
0\end{array}$ \\
\hline Castilla y León & 284,44 & 558,44 & 55,59 & 0,00 & 0,00 & 78,92 & 1144,46 & 0,00 & 51,27 & 0,00 & 396,12 & 15,14 & 0,00 & 808,37 & 0,00 & 0,11 & 3392,85 \\
\hline Galicia & 55,90 & 117,52 & 21,44 & 0,00 & 0,00 & 0,00 & 466,50 & 0,00 & 0,00 & 0,00 & 1352,41 & 0,33 & 0,00 & 0,00 & 0,00 & 0,00 & 2014,10 \\
\hline Andalucía & 23,09 & 157,37 & 50,46 & 110,31 & 30,89 & 0,00 & 457,63 & 0,18 & 256,38 & 41,25 & 185,48 & 1,35 & 7,71 & 613,98 & 9,16 & 3,10 & 1948,32 \\
\hline $\begin{array}{l}\text { Castilla-La } \\
\text { Mancha }\end{array}$ & 22,87 & 182,79 & 24,04 & 0,00 & 2,90 & 0,00 & 855,22 & 1,13 & 27,15 & 4,68 & 165,89 & 6,71 & 1,51 & 361,75 & 0,00 & 0,03 & 1656,68 \\
\hline Aragón & 115,07 & 52,44 & 39,00 & 0,00 & 0,00 & 1,49 & 464,83 & 0,36 & 18,61 & 11,10 & 59,75 & 0,00 & 0,07 & 630,67 & 1,74 & 0,26 & 1395,39 \\
\hline $\begin{array}{l}\text { Comunitat } \\
\text { Valenciana }\end{array}$ & 14,01 & 44,50 & 5,71 & 0,00 & 0,00 & 0,00 & 425,83 & 0,00 & 0,00 & 0,00 & 54,65 & 0,00 & 0,00 & 6,13 & 0,00 & 0,00 & 550,85 \\
\hline $\begin{array}{l}\text { Comunidad Foral } \\
\text { de Navarra }\end{array}$ & 47,22 & 93,81 & 25,50 & 0,00 & 0,00 & 0,00 & 197,61 & 0,00 & 0,00 & 0,00 & 7,36 & 0,00 & 0,00 & 130,47 & 0,00 & 1,15 & 503,13 \\
\hline $\begin{array}{l}\text { Principado de } \\
\text { Asturias }\end{array}$ & 0,35 & 15,28 & 0,00 & 0,00 & 0,00 & 0,00 & 330,71 & 0,00 & 4,63 & 0,00 & 14,91 & 0,00 & 0,00 & 0,00 & 0,00 & 0,00 & 365,88 \\
\hline Catalunya & 46,03 & 47,28 & 29,23 & 0,00 & 0,19 & 0,00 & 107,31 & 0,00 & 0,00 & 33,80 & 0,00 & 0,00 & 0,00 & 41,80 & 0,00 & 7,92 & 313,55 \\
\hline $\begin{array}{l}\text { País Vasco/ } \\
\text { Euskadi }\end{array}$ & 0,00 & 26,38 & 0,00 & 0,00 & 0,00 & 0,00 & 212,02 & 0,00 & 0,00 & 0,00 & 0,00 & 0,00 & 0,00 & 0,00 & 0,00 & 0,00 & 238,40 \\
\hline La Rioja & 0,00 & 100,53 & 12,07 & 0,00 & 0,00 & 0,00 & 48,88 & 0,00 & 4,40 & 0,00 & 18,43 & 0,00 & 0,00 & 9,98 & 0,00 & 0,63 & 194,92 \\
\hline Región de Murcia & 0,00 & 15,07 & 0,06 & 0,00 & 0,00 & 0,00 & 39,33 & 4,40 & 3,90 & 0,00 & 7,96 & 0,00 & 5,03 & 0,00 & 0,00 & 0,00 & 75,75 \\
\hline Cantabria & 0,37 & 2,65 & 0,00 & 0,00 & 0,00 & 10,10 & 17,78 & 0,00 & 0,02 & 0,00 & 0,41 & 0,00 & 0,00 & 0,00 & 0,00 & 0,00 & 31,33 \\
\hline $\begin{array}{l}\text { Total España } \\
\text { (Península) }\end{array}$ & 609,35 & 1414,09 & 263,09 & 110,31 & 33,97 & 90,51 & 4768,11 & 6,06 & 366,35 & 90,83 & 2263,37 & 23,52 & 14,31 & 2603,16 & 10,90 & 13,20 & 12681,15 \\
\hline
\end{tabular}




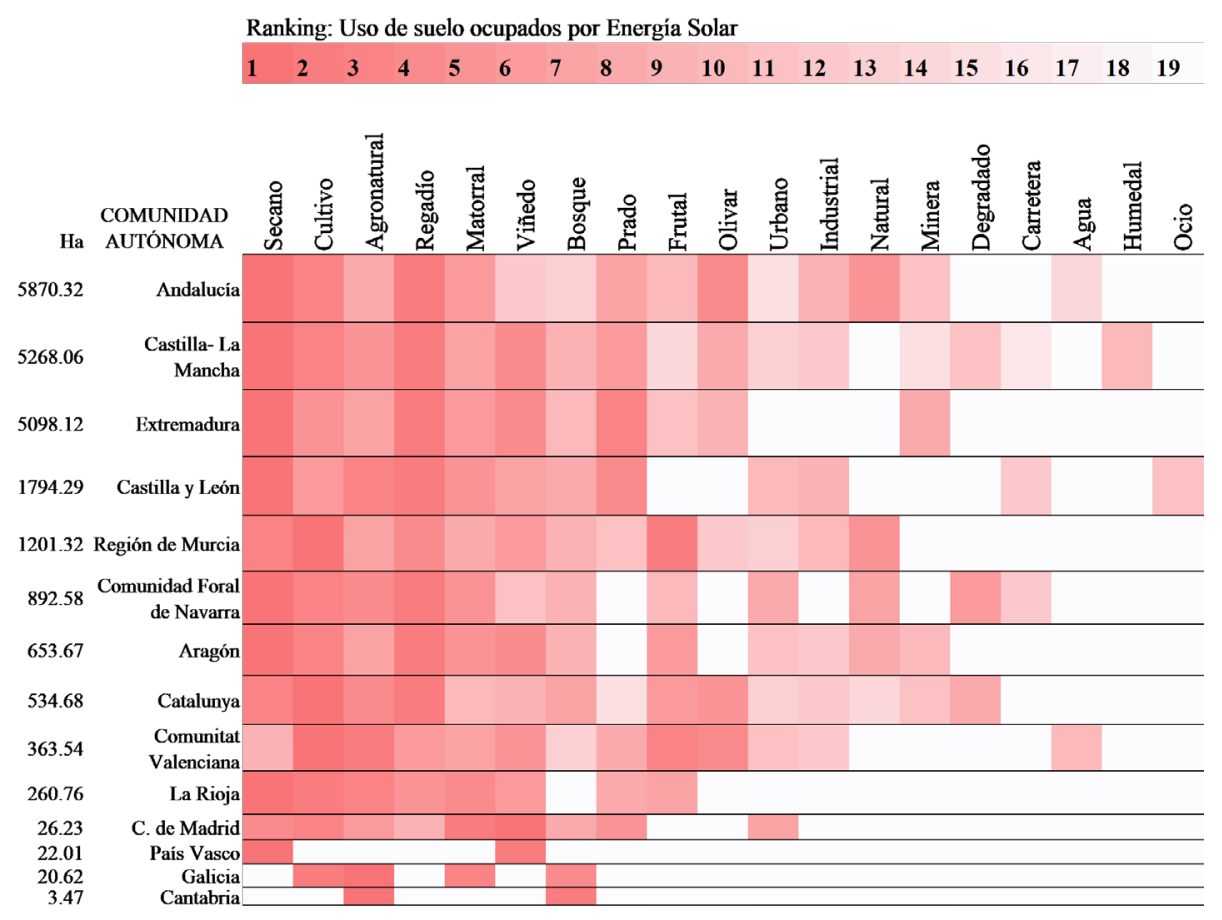

Figura 8. Usos de suelo (2006) ocupados por energía solar (2011). Fuente: CLC 2006 y SIOSE 2011.

Figure 8. Land Uses (2006) covered by solar power farms (2011). Source: CLC 2006 y SIOSE 2011

como las zonas de uso urbano más cercanas a las instalaciones energéticas. En el caso de la energía eólica, tanto en aeropuertos $(1,47 \%)$, como puertos $(0,86 \%)$ y carreteras $(2,73 \%)$, los valores pueden resultar algo significativos si tenemos en cuenta la forma y la extensión que ocupan estas categorías de usos de suelo. Además, en el análisis por Comunidades Autónomas, Aragón y Castilla-León presentan porcentajes superiores al 5\% para el caso de la cercanía a instalaciones eólicas. De este modo, cabe destacar la Comunidad Foral de Navarra, con un $14 \%$ de redes de transporte, identificado como el uso de suelo más cercano a instalaciones de energía solar (figura 10).

Al igual que ocurre con el resto de análisis que se han realizado sobre usos de suelo donde se complementan SIOSE y CLC, no se identifican trabajos similares para establecer algún tipo de comparación valorativa. Sin embargo, la información obtenida en este punto, permiten valorar el alcance y el tipo de análisis que permite realizar la complementación de estas bbdds.

Valoración sobre el uso de SIOSE para el estudio del emplazamiento de las instalaciones de energía solar y eólica sobre espacios naturales protegidos

De la exploración realizada se desprende que en el territorio peninsular español, 67 ha correspondientes a instalaciones de energía solar, se encuentran emplazadas en algún espacio natural, frente a las 1.112,07 ha de instalaciones de energía eólica. Un $0,31 \%$ y un $8,77 \%$ del total, respectivamente (tabla 5 y 6 ).

En el caso de la energía solar, Andalucía con 36 ha, Catalunya con 17,35 ha y la 
Tabla 4

Ha usos de suelo (2006) ocupados por energía solar (2011). Fuente: CLC 2006, SIOSE 2011

Table 4

Land Use hectares (2006) covered by solar power (2011). Source: CLC, 2006; SIOSE, 2011.

\begin{tabular}{|c|c|c|c|c|c|c|c|c|c|c|c|c|c|c|c|c|c|c|c|c|}
\hline $\begin{array}{l}\text { Hectáreas / } \\
\text { Energía Solar }\end{array}$ & 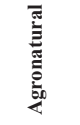 & 嵒 & 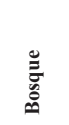 & & $\stackrel{\nexists}{\underline{z}}$ & 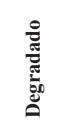 & Eू & 嵒 & 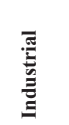 & $\frac{\bar{\pi}}{\frac{5}{2}}$ & 莺 & 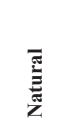 & $\stackrel{\circ}{\ddot{d}}$ & $\frac{\pi}{\partial}$ & $\stackrel{\circ}{\pi}$ & 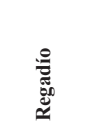 & 竧 & 竎 & 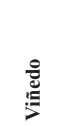 & 竧䒩 \\
\hline Andalucía & 69,54 & 1,29 & 8,54 & 0,00 & 961,67 & 0,00 & 28,71 & 0,00 & 33,41 & 142,74 & 23,82 & 160,87 & 0,00 & 321,82 & 79,77 & $1.080,78$ & $2.939,15$ & 0,02 & 18,20 & $5.870,32$ \\
\hline $\begin{array}{l}\text { Castilla-La } \\
\text { Mancha }\end{array}$ & 224,30 & 0,00 & 5,64 & 0,20 & 867,00 & 2,69 & 0,30 & 4,02 & 0,63 & 62,12 & 0,29 & 0,00 & 0,00 & 8,75 & 63,64 & $1.437,51$ & $2.324,00$ & 0,48 & 266,50 & $5.268,06$ \\
\hline Extremadura & 49,07 & 0,00 & 11,90 & 0,00 & 188,77 & 0,00 & 0,69 & 0,00 & 0,00 & 76,98 & 27,89 & 0,00 & 0,00 & 23,36 & $1.048,11$ & $1.285,69$ & $2.195,98$ & 0,00 & 189,69 & $5.098,12$ \\
\hline $\begin{array}{l}\text { Castilla y } \\
\text { León }\end{array}$ & 139,30 & 0,00 & 5,67 & 0,22 & 51,34 & 0,00 & 0,00 & 0,00 & 3,62 & 61,83 & 0,00 & 0,00 & 0,60 & 0,00 & 123,21 & 209,14 & $1.188,71$ & 0,97 & 9,68 & $1.794,29$ \\
\hline $\begin{array}{l}\text { Región de } \\
\text { Murcia }\end{array}$ & 50,31 & 0,00 & 6,28 & 0,00 & 270,71 & 0,00 & 243,76 & 0,00 & 4,08 & 46,77 & 0,00 & 101,50 & 0,00 & 0,69 & 0,80 & 193,26 & 200,82 & 0,43 & 81,92 & $1.201,32$ \\
\hline $\begin{array}{l}\text { Comunidad } \\
\text { Foral de } \\
\text { Navarra }\end{array}$ & 112,58 & 0,00 & 6,58 & 0,03 & 147,11 & 34,16 & 3,83 & 0,00 & 0,00 & 42,73 & 0,00 & 12,98 & 0,00 & 0,00 & 0,00 & 157,64 & 362,60 & 11,48 & 0,88 & 892,58 \\
\hline Aragón & 9,09 & 0,00 & 2,00 & 0,00 & 46,89 & 0,00 & 17,92 & 0,00 & 0,00 & 19,82 & 0,95 & 8,29 & 0,00 & 0,00 & 0,00 & 60,73 & 442,03 & 0,20 & 45,74 & 653,67 \\
\hline Catalunya & 50,62 & 0,00 & 11,36 & 0,00 & 190,39 & 6,28 & 19,22 & 0,00 & 2,89 & 3,79 & 3,51 & 1,27 & 0,00 & 21,62 & 0,95 & 126,71 & 88,34 & 2,19 & 5,52 & 534,68 \\
\hline $\begin{array}{l}\text { Comunitat } \\
\text { Valenciana }\end{array}$ & 53,66 & 6,23 & 0,58 & 0,00 & 96,57 & 0,00 & 53,02 & 0,00 & 0,59 & 25,09 & 0,00 & 0,00 & 0,00 & 47,50 & 14,44 & 25,87 & 8,16 & 3,24 & 28,59 & 363,54 \\
\hline La Rioja & 25,02 & 0,00 & 0,00 & 0,00 & 27,25 & 0,00 & 4,92 & 0,00 & 0,00 & 14,93 & 0,00 & 0,00 & 0,00 & 0,00 & 0,92 & 12,33 & 167,27 & 0,00 & 8,12 & 260,76 \\
\hline $\begin{array}{l}\text { Comunidad de } \\
\text { Madrid }\end{array}$ & 0,88 & 0,00 & 0,36 & 0,00 & 4,42 & 0,00 & 0,00 & 0,00 & 0,00 & 5,25 & 0,00 & 0,00 & 0,00 & 0,00 & 0,97 & 0,04 & 2,52 & 0,61 & 11,18 & 26,23 \\
\hline $\begin{array}{l}\text { País Vasco/ } \\
\text { Euskadi }\end{array}$ & 0,00 & 0,00 & 0,00 & 0,00 & 0,00 & 0,00 & 0,00 & 0,00 & 0,00 & 0,00 & 0,00 & 0,00 & 0,00 & 0,00 & 0,00 & 0,00 & 20,83 & 0,00 & 1,18 & 22,01 \\
\hline Galicia & 19,29 & 0,00 & 0,19 & 0,00 & 0,94 & 0,00 & 0,00 & 0,00 & 0,00 & 0,20 & 0,00 & 0,00 & 0,00 & 0,00 & 0,00 & 0,00 & 0,00 & 0,00 & 0,00 & 20,62 \\
\hline Cantabria & 3,40 & 0,00 & 0,07 & 0,00 & 0,00 & 0,00 & 0,00 & 0,00 & 0,00 & 0,00 & 0,00 & 0,00 & 0,00 & 0,00 & 0,00 & 0,00 & 0,00 & 0,00 & 0,00 & 3,47 \\
\hline $\begin{array}{l}\text { Total España } \\
\text { (Península) }\end{array}$ & 807,06 & 7,51 & 59,17 & 0,45 & $2.853,05$ & 43,14 & 372,35 & 4,02 & 45,22 & 502,24 & 56,47 & 284,90 & 0,60 & 423,73 & $1.332,82$ & $4.589,69$ & $9.940,41$ & 19,64 & 667,19 & $22.009,66$ \\
\hline
\end{tabular}




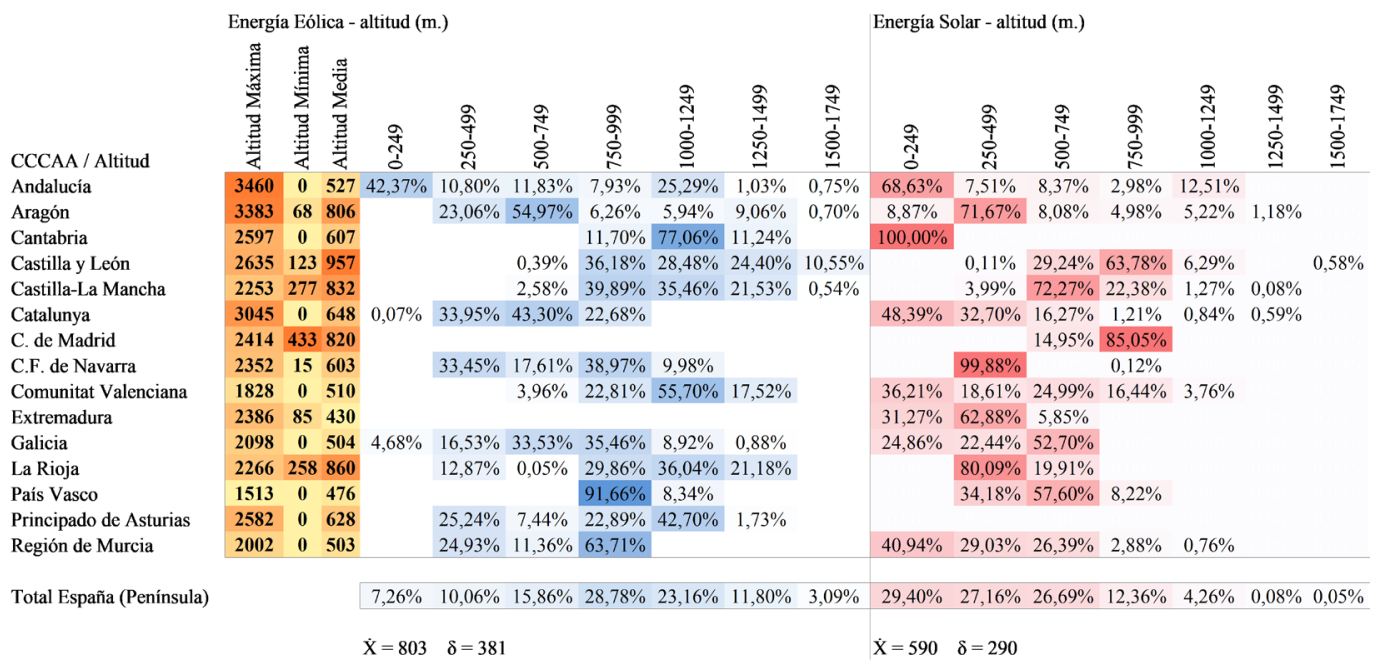

Figura 9. Localización en \% de superficie de energía eólica y solar por CCAA y altitud, 2011. Fuente: SIOSE 2011 y NASA SRTM.

Figure 9. Location of wind and solar energy surface by Autonomous Community and altitude, 2011 (in percentage). Source: SIOSE 2011 y NASA SRTM.

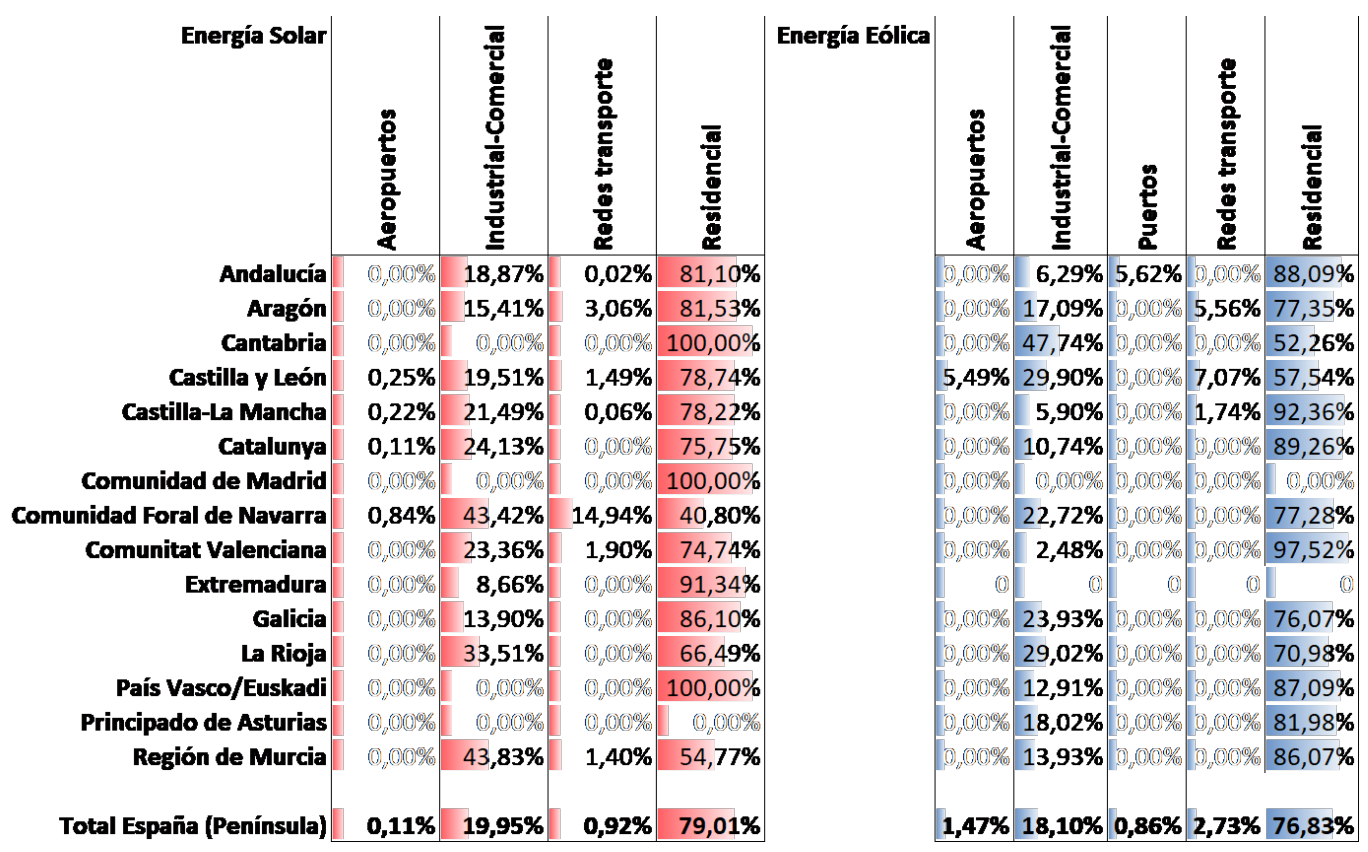

Figura 10. Tipo de suelo urbano más cercano a las instalaciones de energía solar y eólica 2011.

Fuente: SIOSE 2011.

Figure 10. Urban land use category nearer to the solar and wind farms, 2011. Source: SIOSE 2011. 
Tabla 5

Superficie de instalaciones (Ha) de energía eólica en ENP. Fuente: SIOSE 2011 y MAGRAMA (2016).

Table 5

Solar power hectares on Natural Protected Areas. Source: SIOSE 2011 and MAGRAMA (2016).

\begin{tabular}{|c|c|c|c|c|c|c|c|c|c|c|}
\hline EÓLICA & 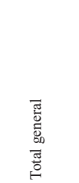 & 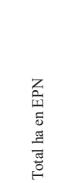 & 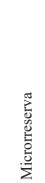 & 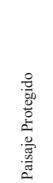 & 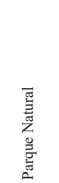 & 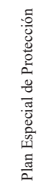 & 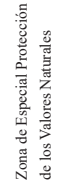 & 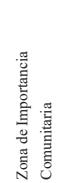 & 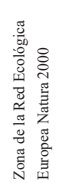 & 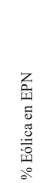 \\
\hline Andalucia & $1.948,32$ & 317,74 & 0,00 & 0,00 & 89,67 & 0,00 & 0,00 & 228,07 & 0,00 & 16,31 \\
\hline Aragón & $1.395,39$ & 0,55 & 0,00 & 0,00 & 0,55 & 0,00 & 0,00 & 0,00 & 0,00 & 0,04 \\
\hline Cantabria & 31,33 & 1,88 & 0,00 & 0,00 & 0,04 & 0,00 & 0,00 & 0,00 & 1,84 & 6,01 \\
\hline Castilla y León & $3.392,85$ & 19,80 & 0,00 & 0,00 & 18,74 & 0,00 & 0,00 & 0,00 & 1,05 & 0,58 \\
\hline Castilla-La Mancha & $1.656,68$ & 0,06 & 0,06 & 0,00 & 0,00 & 0,00 & 0,00 & 0,00 & 0,00 & 0,00 \\
\hline Catalunya & 313,55 & 10,95 & 0,00 & 0,00 & 0,00 & 10,95 & 0,00 & 0,00 & 0,00 & 3,49 \\
\hline C. de Madrid & 0,00 & 0,00 & & & & & & & & 0,00 \\
\hline C.F. de Navarra & 503,13 & 15,78 & 0,00 & 15,74 & 0,05 & 0,00 & 0,00 & 0,00 & 0,00 & 3,14 \\
\hline Comunitat Valenciana & 550,85 & 0,00 & & & & & & & & 0,00 \\
\hline Extremadura & 0,00 & 0,00 & & & & & & & & 0,00 \\
\hline Galicia & $2.014,10$ & 702,37 & 0,00 & 0,00 & 4,87 & 0,00 & 697,50 & 0,00 & 0,00 & 34,87 \\
\hline La Rioja & 195,32 & 0,00 & & & & & & & & 0,00 \\
\hline Región de Murcia & 75,75 & 0,00 & & & & & & & & 0,00 \\
\hline País Vasco & 238,40 & 42,94 & 0,00 & 0,00 & 42,94 & 0,00 & 0,00 & 0,00 & 0,00 & 18,01 \\
\hline $\begin{array}{l}\text { Principado de } \\
\text { Asturias }\end{array}$ & 365,88 & 0,00 & & & & & & & & 0,00 \\
\hline $\begin{array}{l}\text { Total España } \\
\text { (Peninsula) }\end{array}$ & $12.681,15$ & $1.112,07$ & 0,06 & 15,74 & 156,86 & 10,95 & 697,50 & 228,07 & 2,90 & 8,77 \\
\hline
\end{tabular}

Tabla 6

Superficie de instalaciones (Ha) de energía solar en ENP. Fuente: SIOSE 2011, MAGRAMA (2016). Table 6

Solar power hectares on Natural Protected Areas. Source: SIOSE 2011, MAGRAMA (2016).

\begin{tabular}{|c|c|c|c|c|c|}
\hline $\begin{array}{l}\text { CODE } \\
\text { CLC }\end{array}$ & $\begin{array}{l}\text { Nombre } \\
\text { análisis }\end{array}$ & $\begin{array}{l}\text { CODE } \\
\text { CLC }\end{array}$ & $\begin{array}{l}\text { Nombre } \\
\text { análisis }\end{array}$ & CODE CLC & Nombre análisis \\
\hline $\begin{array}{l}111 y \\
112\end{array}$ & Urbano & 211 & Secano & $\begin{array}{l}311,312 y \\
313\end{array}$ & Bosque \\
\hline 121 & Industrial & 212 у 213 & Regadío & 321 & Prado \\
\hline 122 & Carretera & 221 & Viñedo & $\begin{array}{l}322,323 y \\
324\end{array}$ & Matorral \\
\hline $\begin{array}{l}123 y \\
124\end{array}$ & Puerto & 222 & Frutal & $\begin{array}{l}331,332,333 \\
412,421,422 \\
\text { у } 423\end{array}$ & Natural \\
\hline 131 & Minera & 223 & Olivar & 334 & Quemado \\
\hline $\begin{array}{l}132 \mathrm{y} \\
133\end{array}$ & Degradado & 231 & Prado & 335 & Montaña \\
\hline \multirow[t]{3}{*}{$\begin{array}{l}141 \mathrm{y} \\
142\end{array}$} & Ocio & $\begin{array}{l}241,242 \text { y } \\
243\end{array}$ & Cultivo & 411 & Humedal \\
\hline & & 244 & Agronatural & $\begin{array}{l}511,512,521 \\
\text { y } 522\end{array}$ & Agua \\
\hline & & & & 523 & Mar \\
\hline
\end{tabular}


Comunidad Valenciana con casi 8 ha son las que suman casi la totalidad de la superficie con uso solar; y para el caso de la energía eólica, Extremadura con 702,37 ha $y$ Andalucía con 317,74 ha son las que más suelo ubicado en espacios naturales ocupan (tabla 5).

El tipo de protección de los espacios naturales con hectáreas de instalaciones de energía solar corresponde fundamentalmente a la figura de Parque Natural (15,54 ha), Plan Especiales de Protección (17,35 ha) y Zona de Importancia Comunitaria (30,96 ha) (tabla 6). En el caso de las instalaciones de energía eólica, los tipos de espacio natural más ocupados por estas instalaciones son Zona de Especial Protección de los Valores Naturales (697,50 ha), Zona de Importancia Comunitaria $(228,07$ ha) y Parque Natural (156,86 ha) (tabla 5).

\section{Conclusiones}

Este trabajo ha cubierto su objetivo principal que era el de explorar y valorar las facultades de la base de datos SIOSE para realizar diversos análisis espaciotemporales sobre las instalaciones de energía renovable solar y eólica. Cabe destacar que SIOSE tiene la capacidad de reflejar la localización y extensión de las instalaciones, sin embargo, el principal inconveniente subyace en la dificultad para establecer los límites concretos de las mismas cuando estas se encuentran recogidas en polígonos compuestos. Este hecho condiciona esta base de datos geográfica para realizar análisis espaciales explícitos sobre cambios de cobertura de suelo entre distintos periodos temporales. Sin embargo, como se ha mostrado en este trabajo, utilizando otras fuentes como CORINE Land Cover (CLC), se pueden llevar a cabo análisis de cambio de usos de suelo referidos a coberturas anteriores concretas (usos de suelo CLC) respecto a ocupación posterior por usos de suelo para la producción de energía solar y eólica (SIOSE).
SIOSE permite, como se muestra en este estudio, la posibilidad de realizar análisis de localización y evolución temporal en extensión de las instalaciones solar y eólica por entidades administrativas, de patrones de proximidad respecto a otros usos de suelo o actividades (residencial, industrial, aeropuertos...), de patrones de localización respecto a la altitud; $y$, como se ha señalado, de patrones de ocupación respecto a otros usos y coberturas de suelo.

Los análisis realizados en el territorio español (península) han permitido contabilizar el enorme incremento experimentado por la implantación de estas instalaciones de energía renovable solar y eólica entre 2005 y 2011, destacando el enorme incremento de la energía solar (de 689 a 22.000 ha). Permitiendo además observar las diferencias entre las distintas comunidades autónomas.

Con respecto a la ocupación de usos de suelo por la actividad de producción de energía eólica y solar, se pudieron constatar algunos patrones de localización general, como la ubicación preferente de la energía eólica, sobre áreas de matorral, bosque, prado y espacios agronaturales, frente a la localización sobre cultivos de secano de la energía solar. Si bien es cierto que se observaron muchos otros matices, así como diferencias por comunidades autónomas. También en función del grado de desarrollo que han alcanzado la implantación de esta energía en cada región.

Muchos de estos patrones de localización que se han mostrado en este trabajo podrían haberse esgrimido de manera hipotética, como la diferencia de ubicación o la cota media de localización de la energía solar, o que de manera general, sean las áreas residenciales el uso más próximo a la mayoría de las instalaciones energéticas, no obstante, SIOSE, con el apoyo de CLC puede permitir corroborar estas hipótesis, además de dar cuenta de sus posibilidades para realizar estudios de mayor alcance. 
Los ejercicios de valoración que se han desarrollado en este trabajo, demuestran que SIOSE complementado con otras bbdds geográficas sirven de base para responder distintas cuestiones que se pueden plantear sobre el desarrollo de la implantación territorial de energía renovable solar y eólica en el caso concreto de España.

Este trabajo también pone de manifiesto la necesidad de continuar desarrollando este tipo de bbdds de UCS, mejorando la precisión y el detalle en la cartografía de los parques solares y eólicos, ya que es previsible que continúe el rápido incremento en el empleo de energías limpias en consonancia con el abaratamiento de las tecnologías en la producción de las mismas y el agotamiento e impacto ambiental de los combustibles fósiles (IPCC, 2011).

\section{Agradecimientos}

Esta investigación se enmarca en el proyecto COMPLEX del séptimo marco de la Comisión Europea.

\section{Referencias}

Almonacid, A. \& Nahuelhual, L. (2009). Estimación del potencial eólico y costos de producción de energía eólica en la costa de Valdivia, Sur de Chile. Agro sur, 37(2), 103109. https://doi.org/10.4206/agrosur.2009. v37n2-04

Alonso, P. M., Hewitt, R., Pacheco, J. D., Bermejo, L. R., Jiménez, V. H., Guillén, J. V., ... de Boer, C. (2016). Losing the roadmap: Renewable energy paralysis in Spain and its implications for the EU low carbon economy. Renewable energy, 89, 680-694. http:// dx.doi.org/10.1016/j.renene.2015.12.004

Amarante, O. A., Brower, M., Zack, J., \& Sá, A. (2001). Atlas do potencial eólico brasileiro. Brasilia: Ministerio de Minas e EnergiaEletrobras. Recuperado de http://www.cresesb.cepel.br/publicacoes/ download/atlas_eolico/atlas $\% 20 \mathrm{do} \% 20$ potencial $\% 20$ eolico $\% 20$ brasileiro.pdf
Aymamí, J., García, A., Lacave, O., Lledó, L., Mayo, M., \& Parés, S. (2011). Análisis del recurso. Atlas eólico de España (Estudio Técnico PER 2011-2020) Madrid: IDAE. Recuperado de http://www.idae.es/ uploads/documentos/documentos_11227 e4_atlas_eolico_A_9b90ff10.pdf

Castro, M. de, Martín-Vide, J., \& Alonso, S. (2005). El clima de España: Pasado, presente y escenarios de clima para el siglo $X X I$.España.Ministeriode MedioAmbiente. ISBN 84-8320-303-0. Recuperado de https://digital.csic.es/britstream/ 10261/35782/1/09047/1228000f24f_ tcm7-12417.pdf

Couture, T. \& Gagnon, Y. (2010). An analysis of feed-in tariff remuneration models: Implications for renewable energy investment. Energy policy, 38(2), 955-965. https://doi.org/10.1016/j. enpol.2009.10.047

Comisión Europea (2011). Energy roadmap 2050. Luxembourg: Publications office of the European Union. ISBN 97892-79-21798-2. Recuperado de https:// ec.europa.eu/energy/sites/ener/files/ documents/2012_energy_roadmap_2050_ en_0.pdf

Corine Land Cover [CLC] (2006). Programa Copernicus. Comisión Europea. Recuperado de https://www.eea.europa.eu/ data-and-maps/data/clc-2006-raster

Celik, A. N., Muneer, T., \& Clarke, P. (2009). A review of installed solar photovoltaic and thermal collector capacities in relation to solar potential for the EU-15. Renewable energy, 34(3), 849-856. http://dx.doi.org/10.1016/j. renene.2008.05.025

Chang, J., Leung, D. Y., Wu, C. Z., \& Yuan, Z. H. (2003). A review on the energy production, consumption, and prospect of renewable energy in China. Renewable and sustainable energy reviews, 7(5), 453-468. http://dx.doi.org/10.1016/S13640321(03)00065-0 
Del Río, P. \& Gual, M. A. (2007). An integrated assessment of the feed-in tariff system in Spain. Energy policy, 35(2), 994-1.012. https://doi.org/10.1016/j. enpol.2006.01.014

Del Río, P. (2008). Ten years of renewable electricity policies in Spain: An analysis of successive feed-in tariff reforms. Energy policy, 36(8), 2.917-2.929. https://doi. org/10.1016/j.enpol.2008.03.025

Del Río, P. \& Mir-Artigues, P. (2012). Support for solar PV deployment in Spain: Some policy lessons. Renewable and sustainable energy reviews, 16(8), 5.557-5.566. https://doi.org/10.1016/j. rser.2012.05.011

Díaz-Pacheco, J. \& Gutiérrez, J. (2014). Exploring the limitations of CORINE Land Cover for monitoring urban landuse dynamics in metropolitan areas. Journal of land use science, 9(3), 243259. http://dx.doi.org/10.1080/174742 3X.2012.761736

Dinica, V. (2008). Initiating a sustained diffusion of wind power: the role of publicprivate partnerships in Spain. Energy policy, 36(9), 3562-3571. https://doi.org/10.1016/j. enpol.2008.06.008

Espejo, C. (2004). La energía eólica en España. Investigaciones geográficas, $35, \quad 45-65$. https://doi.org/10.14198/ ingeo2004.35.03

Espejo, C. (2010). Los nuevos paisajes de la energía solar: las centrales termosolares. Nimbus, 25-26,65-91.Recuperado dehttps:// core.ac.uk/download/pdf/143457590.pdf

Evans, A., Strezov, V., \& Evans, T. J. (2009). Assessment of sustainability indicators for renewable energy technologies. Renewable and sustainable energy reviews, 13(5), 1.082-1.088. http://dx.doi.org/10.1016/j. rser.2008.03.008

Faulín, J., Lera, F., Pintor, J. M., \& García, J. (2006). The outlook for renewable energy in Navarre: an economic profile. Energy policy, 34(15), 2.201-2.216. https://doi. org/10.1016/j.enpol.2005.04.005

González-Ávila, M. E., Beltrán-Morales, L. F., Troyo-Diéguez, E., \& Ortega-Rubio, A. (2006). Potencial de aprovechamiento de la energía eólica para la generación de energía eléctrica en zonas rurales de México. Interciencia, 31(4), 240-245. Recuperado de http://www.redalyc.org/ articulo.oa? $\mathrm{id}=33911502$

Hewitt, R. J., Winder, N. P., Jiménez, V. H., Alonso, P. M., \& Bermejo, L. R. (2017). Innovation, pathways and barriers in Spain and beyond: an integrative research approach to the clean energy transition in Europe. Energy research \& social science, 34, 260-271. https://doi.org/10.1016/j. erss.2017.08.004

Ignateva, M. F. \& Pérez, B. P. (2008). El desarrollo de las energías renovables y el paisaje: algunas bases para la implementación de la Convención Europea del Paisaje en la política energética española. Cuadernos geográficos, 43, 289309. Recuperado de http://www.redalyc. org/articulo.oa?id=17104314

Ignateva, M. F. (2010). Los paisajes de la energía eólica: su percepción social y gestión en España. Nimbus, 25-26, 93110. Recuperado de https://core.ac.uk/ download/pdf/143456161.pdf

Intergovernmental Panel on Climate Change. (2011). Fuentes de energía renovables y mitigación del cambio climático. (Resumen para responsables de políticas. Informe del Grupo de trabajo III del Grupo Intergubernamental de expertos sobre el Cambio Climático (IPCC) y Resumen técnico Informe aceptado por el Grupo de trabajo III del IPCC pero no aprobado en detalle). Ginebra, Suiza. ISBN 978-92-9169-331-3. Recuperado de https:// www.ipcc.ch/pdf/special-reports/srren/ srren_report_es.pdf

Jäger-Waldau, A. (2007). Photovoltaics and renewable energies in Europe. Renewable and sustainable energy reviews, 11(7), 1.414-1.437. http://dx.doi.org/10.1016/j.rser.2005.11.001 
Olcina Cantos, J. (1994). Riesgos climáticos en la Península Ibérica. Madrid: Libros penthalon. Acción divulgativa, S.L. ISBN: 978-8479550721

Larsen, J. K. \& Madsen, J. (2000). Effects of wind turbines and other physical elements on field utilization by pink-footed geese (Anser brachyrhynchus): A landscape perspective. Landscape ecology, 15(8), 755-764. https:// doi.org/10.1023/A:1008127702944

Martín Vide, J. \& Olcina Cantos, J. (2001). Climas y tiempos de España. Madrid: Alianza editorial. ISBN: 9788420657776

Rabus, B., Eineder, M., Roth, A., \& Bamler, R. (2003). The shuttle radar topography mission-a new class of digital elevation models acquired by spaceborne radar. ISPRS journal of photogrammetry and remote sensing, 57(4), 241-262. http://dx.doi. org/10.1016/S0924-2716(02)00124-7

Red Eléctrica de España (REE) (2017). Las energías renovables en el sistema eléctrico español 2016. Recuperado de https://www.ree.es/sites/default/ files/11_PUBLICACIONES/Documentos/ Renovab̄les-2016-v2.pdf

Sánchez, Á. C., García, M. F., \& Saguar, P. F. (2011). Análisis económico y medioambiental del sector eléctrico en España. Estudios de economía aplicada, 29(2), 493-514. Recuperado de http://www. redalyc.org/html/301/30120840004/

SIOSE (2005) Sistema de información sobre ocupación del suelo de España. Instituto Geográfico Nacional, Ministerio de Fomento. Recuperado de http://www. siose.es/siosetheme-theme/documentos/ pdf/doc_tec_siose2011_v1.1.pdf
SIOSE (2011). Sistema de Información sobre Ocupación del Suelo de España. Instituto Geográfico Nacional, Ministerio de Fomento. Recuperado de http://www. siose.es/SIOSEtheme-theme/documentos/ pdf/Doc_tec_SIOSE2011_v1.1.pdf

Sözen, A., Arcaklioğlu, E., \& Özalp, M. (2004). Estimation of solar potential in Turkey by artificial neural networks using meteorological and geographical data. Energy conversion and management, 45(18), 3.033-3.052. http://dx.doi. org/10.1016/j.enconman.2003.12.020

Tena, J.C.M. (2011). SIOSE Valencia 2005: Resultados, aplicaciones y comparación con CORINE. Cuadernos de geografía, 89, 1-22. Recuperado de http://roderic.uv.es/ handle/10550/31512

Villa, G., Arozarena, A., del Bosque, I., Valcárcel, N., García, C., \& Solís, M. J. (septiembre, 2005). El plan nacional de observación del territorio en España. Trabajo presentado en XI Congreso nacional de teledetección, Puerto de la Cruz. Tenerife. Recuperado de http://www. aet.org.es/congresos/xi/ten56.pdf

Zografos, C. \& Saladié, S. (2012). La ecología política de conflictos sobre energía eólica. Un estudio de caso en Cataluña. Documents d'anàlisi geogràfica, 58(1), 177-192. Recuperado de https://www.raco. cat/index.php/DocumentsAnalisi/article/ view/250755 